\title{
Immunological Effects of Histotripsy for Cancer Therapy
}

\author{
Alissa Hendricks-Wenger ${ }^{1,2,3}$, Ruby Hutchison ${ }^{3}$, Eli Vlaisavljevich ${ }^{1,3,4}$ \\ and Irving Coy Allen ${ }^{1,2,4,5 *}$
}

${ }^{1}$ Graduate Program in Translational Biology, Medicine and Health, Virginia Tech, Roanoke, VA, United States, ${ }^{2}$ Department of Biomedical Sciences and Pathobiology, Virginia-Maryland College of Veterinary Medicine, Blacksburg, VA, United States, ${ }^{3}$ Department of Biomedical Engineering and Mechanics, Virginia Tech, Blacksburg, VA, United States, ${ }^{4}$ Institute for Critical Technology and Applied Sciences Center for Engineered Health, Virginia Tech, Blacksburg, VA, United States, ${ }^{5}$ Department of Basic Science Education, Virginia Tech Carilion School of Medicine, Roanoke, VA, United States

\section{OPEN ACCESS}

Edited by:

Nurit Hollander,

Tel Aviv University, Israel

Reviewed by:

Tatiana Khokhlova,

University of Washington,

United States

Siqi Guo,

Old Dominion University,

United States

*Correspondence: Irving Coy Allen icallen@vt.edu

Specialty section: This article was submitted to

Cancer Immunity and Immunotherapy, a section of the journal

Frontiers in Oncology

Received: 16 March 2021

Accepted: 10 May 2021

Published: 31 May 2021

Citation: Hendricks-Wenger A, Hutchison R, Vlaisavljevich E and Allen IC (2021) Immunological Effects of Histotripsy for Cancer Therapy. Front. Oncol. 11:681629. doi: 10.3389/fonc.2021.681629
Cancer is the second leading cause of death worldwide despite major advancements in diagnosis and therapy over the past century. One of the most debilitating aspects of cancer is the burden brought on by metastatic disease. Therefore, an ideal treatment protocol would address not only debulking larger primary tumors but also circulating tumor cells and distant metastases. To address this need, the use of immune modulating therapies has become a pillar in the oncology armamentarium. A therapeutic option that has recently emerged is the use of focal ablation therapies that can destroy a tumor through various physical or mechanical mechanisms and release a cellular lysate with the potential to stimulate an immune response. Histotripsy is a non-invasive, non-ionizing, non-thermal, ultrasound guided ablation technology that has shown promise over the past decade as a debulking therapy. As histotripsy therapies have developed, the full picture of the accompanying immune response has revealed a wide range of immunogenic mechanisms that include DAMP and anti-tumor mediator release, changes in local cellular immune populations, development of a systemic immune response, and therapeutic synergism with the inclusion of checkpoint inhibitor therapies. These studies also suggest that there is an immune effect from histotripsy therapies across multiple murine tumor types that may be reproducible. Overall, the effects of histotripsy on tumors show a positive effect on immunomodulation.

Keywords: abscopal effect, histotripsy, focused ultrasound, immunomodulation, ablation

\section{INTRODUCTION}

Although cancer has plagued mankind throughout history, there remains a pressing need to improve treatment options. The core pillars of cancer therapy are chemotherapy, surgery, radiation, immunotherapy, and ablation. The most common treatment option for solid tumors remains surgical resection, even though many patients are not candidates due to tumor size, location, or disease progression (1-4). Tumor ablation modalities have been developed as minimally and noninvasive adjuvants or alternatives to surgery. These procedures include radiofrequency ablation (RFA), microwave ablation, cryoablation, irreversible electroporation (IRE), high-intensity focused 
ultrasound (HIFU), and histotripsy. While these procedures address many of the issues with traditional surgery, there are still many areas where these modalities can be improved.

While the goal of the field of oncology research is to find curative therapies, novel cancer therapies frequently aim for non-curative endpoints such as increased progression free survival, increased overall survival, decreased tumor burden, and improved quality of life. In many cases, the therapeutic goals of local ablation therapy also include a decrease in tumor burden, enhancement of drug delivery to tumors, and the modulation of the immune system. While immune system modulation offers the most promise in terms of long-term patient benefit, it is currently the least predictable and understood benefit of local tumor ablation therapy. The most observable and reliable benefit of local tumor ablation modalities is currently considered to be the decrease in targeted tumor size (5). This can lead to improved patient outcomes by debulking tumors, which can reduce the patient's overall tumor burden, improve local vasculature compression, and reduce tumor nutrient utilization.

\section{THE ROLE OF ABLATION IN IMMUNOMODULATION}

Given that one of the most debilitating aspects of cancer is the burden brought on by metastatic disease, an ideal treatment protocol would address not only debulking larger or debilitating tumors, but also circulating tumor cells and distant metastases. Once cancer has spread from its primary site, focal debulking of individual tumors are less likely to be capable of being a curative treatment option. Therefore, an ideal debulking therapy would not only reduce the size, or fully eliminate, targeted tumors, but would also stimulate the immune system to seek and destroy metastatic lesions throughout the body. This phenomenon has been described as the abscopal effect (Figure 1). There is increasing evidence that immune system activation and systemic tumor elimination is critically important for the elimination of micrometastatic lesions distal to the locally treated tumor, that may not be detectable at the time of treatment or surgery. This review briefly introduces the immune response to non-histotripsy tumor ablation therapies in order to provide context and reference to better understand the state of histotripsy knowledge. More extensive reviews of the immune response to non-histotripsy ablation modalities have been previously conducted at depths that are beyond the scope of this review $(6,7)$.

Before the abscopal effect was formally defined, it was first noted by physicians at the turn of the $20^{\text {th }}$ century who reported that local, targeted radiation of the spleen in leukemia patients would cause changes in the patient's bone marrow leading to the remission of disease $(8,9)$. These clinical findings in combination with many mouse studies led to the abscopal effect being defined by the early 1950's to encompass the effects to distant organs or cancers after applying ionizing radiation (10). This effect was originally thought to only occur for leukemia, where the radiation of the spleen would reduce or eliminate cancerous cells in the body $(10,11)$. However, over the subsequent decades, additional reports of solid tumors displaying this effect periodically emerged. For instance, the reduction of the sizes of distant metastases were reported after the radiation of a single giant follicular lymphoma or a non-Hodgkin's lymphoma tumor $(12,13)$. However, due to the scarcity of reports, the abscopal effect in response to ionizing radiation of solid tumors, including renal cell carcinoma, mammary carcinoma, and neuroblastoma, was frequently dismissed and remained controversial for decades (14-17). To this day, the abscopal effect associated with radiation therapy remains controversial (18). More recently, the development of non-ionizing ablation therapies has led to an increase in the number of abscopal effects reported. Thermal ablations, the first ablation therapies that were developed and
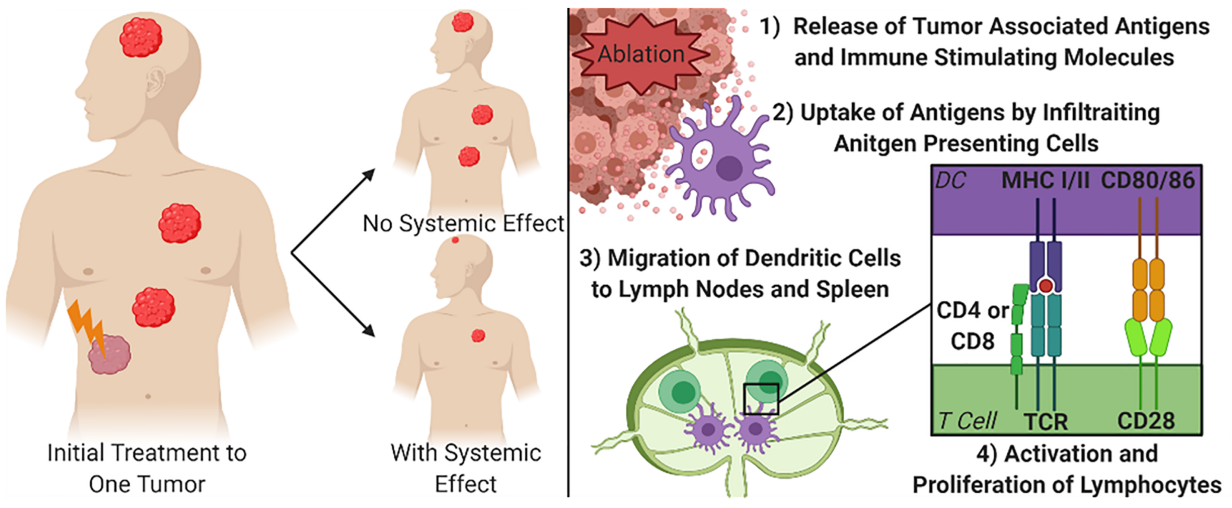

FIGURE 1 | Focal Treatment of Targeted Tumor and Mechanism for Systemic Tumor Control. The right diagram demonstrates the principal of the abscopal effect, where the treatment of one tumor can cause other tumors in the body to shrink or be eliminated to varying degrees due to immune system engagement. The left diagram depicts the simplified mechanism for achieving a systemic effect from focal therapies. DC, Dendritic cell; MHC, Major Histocompatibility Complex; TCR, T cell receptor. 
widely accepted, also have reported sporadic cases of a systemic immune mediated effect where untreated, distal metastases were found to spontaneously regress $(14,19,20)$. One of these earlier case studies showed that the treatment of a primary renal cell carcinoma tumor with RFA caused a spontaneous regression of pulmonary metastases (14). On the other hand, as more nonthermal ablation modalities are being developed pre-clinical studies have had more promising correlations between focal ablation therapy and the systemic effects (21-23). In general, even though there have been promising trends that these therapies can offer systemic therapeutic effects for patients, it should be noted that the reproducibility between patients, tumor types, and therapy modalities is less than optimal (6).

To achieve an abscopal effect, it is not enough to simply release non-specific markers of tissue damage or cell death, if that were the case surgical resections would stimulate systemic effects. Instead, there is a need to release specific tumor associated antigens that allow the immune system to build a targeted response (Figure 1). When focused, local ablation therapies break up the stromal tissues of a tumor this can create an increased opportunity for immune cells to physically access the tumor $(24,25)$. As the cancer cells are ablated, there is an increased abundance accessible tumor antigens that can be recognized by infiltrating antigen presenting cells (APC), such as dendritic cells $(20,26)$. These APCs can in turn activate lymphocytes to guide a more precise immune response. Locally, activation of cytotoxic CD8+ T cells can increase the clearance of any surviving cancer cells within the area treated with the tumor ablation modality (27). For the strongest systemic immune effects, after ablation, dendritic cells will process the available tumor associated antigens and migrate to the nearest draining lymph nodes and the spleen. Once in the lymphoid tissues, the dendritic cells present the antigens through $\mathrm{MHC}$ class I or class II receptors to naïve CD4+ and CD8+ T cells, respectively, along with co-stimulation of CD28 with CD80/86. Studies that show an increase in tumor specific lymphocytes in secondary lymphoid organs or in peripheral blood have also established the correlation between these activated cells and systemic decreases in tumor burden (28-31). More recently, additional studies have also defined $\mathrm{B}$ cell activation, the maintenance of plasma cells, and even the generation of tumor specific antibodies following local tumor ablation and dendritic cell activation $(29,31)$. For example, a study treating hepatocellular carcinoma with RFA reported that only 91 of the 178 patients that had a decreased neutrophil-to-lymphocyte ratio, which is an often used biomarker of systemic immune system activation, independently correlated to a significantly increased survival when compared to patients that did not see the increased ratio (32). While the activation of $\mathrm{T}$ and $\mathrm{B}$ lymphocytes is a core feature of the systemic effects of local tumor ablation therapy, there are many inconsistencies. Similarly, in a prospective study investigating the long-term effects of RFA after treating secondary liver tumors, it was found that only 6 of 49 patients studied had increased antibodies, CD4+ T cells, and/or CD8+ T cells months after treatment (29). While the exact mechanisms underlying the inconsistent reports of the abscopal effect and systemic antitumor immune responses are yet to be fully defined, changes in the local tumor microenvironment have been postulated to significantly impact treatment success.

Although there any many promising pre-clinical and clinical studies showing that ablation therapies have positive effects on systemic immune response, there have also been reports of the opposite. Hepatic RFA and microwave ablation have both seen pre-clinical reports of the treatment of hepatocellular carcinoma tumors and colorectal metastases stimulating growth of existing and new tumors $(33,34)$. There has been multiple hypothesis investigated to explain this phenomenon. One main hypothesis to explain this phenomenon focuses on the subablative hyperthermia that effects margin zone tissues. Studies investigating this effect have shown upregulation of inflammatory pathways in this region, including the IL-6-HGF/c-Met-STAT3VEGF axis and the HSP70 related pathways (35-37). One of these studies also demonstrated that this systemic effect is minimized when the RFA is applied to the healthy liver of rats baring mammary adenocarcinoma tumors at a higher energy dose over a shorter time in order to reduce the damage to surrounding tissues and when administered in combination with HSP inhibitors (35). Another cause associated with increased tumor growth after the application of a minimally invasive ablation, unrelated to changes in the inflammatory response, is the seeding that can occur along the needle track (38).

\section{The Tumor Microenvironment and Tumor Associated Immune Cells}

To determine the cause of systemic anti-tumor immunity, and therefore how to modulate it, there is a need to understand what is occurring within the tumor microenvironment. Within the heterogeneous cell populations of most tumors, there are a variety of tumor-associated immune cells. In an ideal case, these leukocytes are eliminating malignant cells. However, in the case of many cancers, the leukocytes are reprogrammed, or polarized, to support tumor escape and function to promote tumorigenesis through the generation of a relatively immunosuppressive tumor microenvironment. These tumors are often referred to as being immunosuppressive, or immunologically "cold," due to their lack of inflammation and active immune suppression. With multiple types of pro-tumor immune cells that produce factors that can improve the tumor niche, it is difficult for the pro-inflammatory/anti-tumor immune response to overcome this "cold" environment.

The leukocytes most commonly associated with these antiinflammatory and tumor promoting properties include tumorassociated macrophages (TAM), myeloid-derived suppressor cells (MDSC), and tumor-associated neutrophils (TAN) (Figure 2). TAMs are generally defined as CD45+ Ly6C $\mathrm{MHCII}^{+} \mathrm{CD} 1 \mathrm{~b}^{+}(39,40)$. TAMs are also broken down into subsets based on polarization to the M1 macrophage-like phenotype (pro-inflammatory/anti-tumor) $\mathrm{CD}^{+} 8^{+} \mathrm{CD} 80 / 86^{+}$ $\mathrm{CD}_{11 \mathrm{c}^{+}} \mathrm{iNOS}^{+}$and the M2 macrophage-like phenotype (protumor) $\mathrm{CD}_{163}{ }^{+} \mathrm{CD}_{204^{+}} \mathrm{CD} 206^{+} \mathrm{Arg}^{+}$, with M2-like TAMs being the most immunosuppressive $(41,42)$. It should be noted 


\section{PRO-TUMOR IMMUNE CELLS}

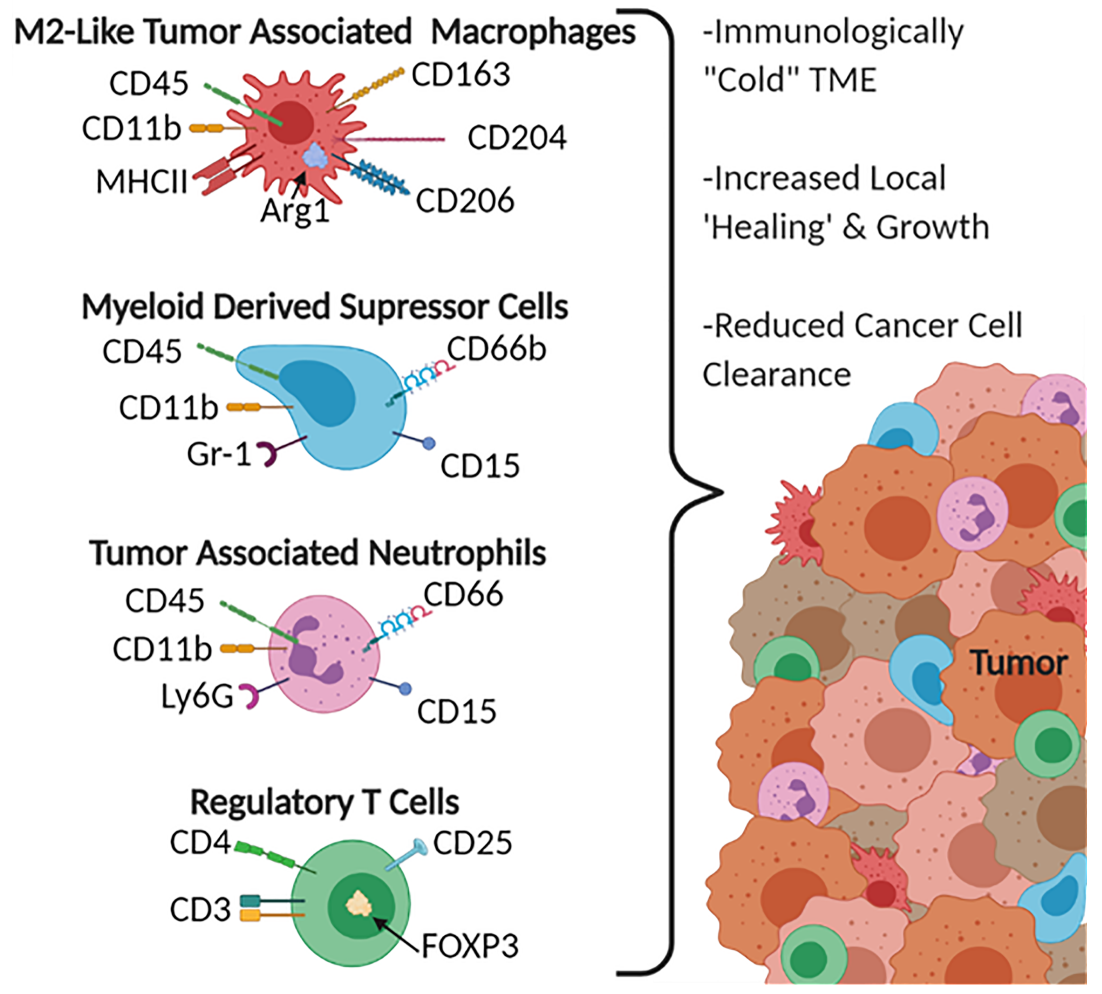

FIGURE 2 | Pro-Tumor Immune Cells. CD66, CD66b, and CD15 are human specific markers. Gr-1 represents both Ly6C and Ly6G, therefore does not distinguish the MDSC subtypes, MDSCs are monocytic when Ly6C+ and granulocytic when Ly6G+. Most of the markers available for pro-tumor immune cells are surface receptors and ligands, exceptions listed here include the intracellular protein Arg1 and the transcription factor FOXP3.

that while the M1-like and M2-like TAMs do share similar expression patterns and activities, they are not the same as the traditional M1 and M2 polarized macrophages. MDSCs are

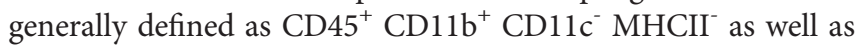
$\mathrm{CD}_{15}{ }^{+}$and $\mathrm{CD}_{6} \mathrm{~b}^{+}$in humans (43-45). MDSCs are broken into two main subtypes: monocytic MDSCs Ly6C ${ }^{+} \mathrm{Ly}^{-}$and granulocytic MDSCs Ly6C $\mathrm{Ly}^{-} \mathrm{G}^{+}$(43). When generally searching for MDSCs, the GR-1 antibody has been used given that it binds to both Ly6C and Ly6G (43). TANs are defined as $\mathrm{CD}_{4} 5^{+} \mathrm{CD}_{11 \mathrm{~b}^{+} \mathrm{Ly}_{6 \mathrm{G}}{ }^{+} \text {and have been reported to express CD66 }}$ and CD15 in human cancers (46-48). It should be noted that the markers that have been established for TANs are indistinguishable from traditional neutrophils, with the most established phenotypic difference between the two cell groups being that neutrophils have a half-life of 6-8 hours whereas TANs have a significantly increased lifespan (an additional 12-24 hours) caused by an inhibition of apoptotic pathways and support from cytokines within the tumor microenvironment (48-52). Similar in nomenclature to TAMs, TANs can also be divided into $\mathrm{N} 1$ pro-inflammatory and $\mathrm{N} 2$ pro-tumor subtypes, however due to limitations in markers there is not an established panel for differentiating N1 from N2 TANs (46).
These anti-inflammatory leukocytes are typically distributed throughout the tumor and margins (Figure 2) and function beyond simply being tumor associated. These leukocytes are frequently referred to as being pro-tumor given that the range of their activities can actively promote tumor growth and development. These functions include reducing the impact of anti-tumor immune responses, recruiting additional tumor supporting cells, helping the tumor grow by stimulating angiogenesis and secreting growth factors, and assisting in the epithelial-to-mesenchymal transition (53-55). For TAMs, this includes producing anti-inflammatory mediators such as IL-10, Arg1, and TGF- $\beta$ (56-58), promoting the expression of checkpoint inhibitors to suppress $\mathrm{T}$ cells $(56,57)$, inducing cancer stem cell proliferation via IL-6 signaling through STAT3 (59), and producing VEGF to stimulate angiogenesis $(60,61)$. These effects are also seen in TANs and MDSCs, which have both been shown to produce similar profiles of cytokines, checkpoint inhibitors, and growth factors (47, 48, 62-64).

In addition to these monocyte and granulocyte derived cells, lymphocytes can also aid in maintaining this "cold" tumor microenvironment. Regulatory $\mathrm{T}$ cells, defined as $\mathrm{CD}^{+} \mathrm{CD}^{+}$ $\mathrm{CD} 25^{+} \mathrm{FOXP}^{+}$, can suppress the adaptive immune response 
(65). While MDSCs, TANs, and TAMs are commonly identified as tumor promoting cells with specific markers and functions, the role of $\mathrm{T}$ regulatory cells is more situationally specific, and their presence and ratio are not necessarily a sign of an immunosuppressive tumor microenvironment. In most cases, the presence of a high ratio of $\mathrm{T}$ regulatory cells, known for inhibiting the function of other $\mathrm{T}$ cells, is a sign of an immunosuppressive tumor microenvironment. Clinically, in ovarian carcinoma it has been established that the recruitment of $\mathrm{T}$ regulatory cells into the tumor stroma leads to decreased survival of patients (66). Additionally, for pancreatic cancer, the $T$ regulatory cells have been found to aid in the pro-carcinogenic inflammation driven by $\mathrm{T}$ helper 17 cells (67). These poor prognostic correlations in patients is tied to the fact that $\mathrm{T}$ regulatory cells can suppress the function of $\mathrm{T}$ helper cells through the production of immunosuppressive cytokines, including IL-10 and TGF- $\beta(68,69)$, secrete perforins and granzymes to directly destroy effector $\mathrm{T}$ cells and B cells (70), and express the checkpoint inhibitor CTLA-4 to suppress APC activity (71). There have also been reports of $\mathrm{T}$ regulatory cells directly increasing the growth of tumors through the stimulation of cancer stem cells via the NF-KB-IL6-STAT4 signaling axis (72).

On the other hand, there are reports of certain cancers, such as colorectal cancer, which have worse prognoses for patients that have a reduced presence of $\mathrm{T}$ regulatory cells within their tumors $(73,74)$. A proposed mechanism for the seemingly contradictory immune promoting function of $\mathrm{T}$ regulatory cells in colorectal cancer is associated with the location of these tumors. In the gastrointestinal tract, T regulatory cells are primed against the microbiota of the intestinal space instead of the cancer (74). This secondary, non-tumoral target for the immune system allows for the immune response to become more robust in the colon, which can in turn destroy the cancerous cells, without being hampered by the immunosuppressive control of the tumor microenvironment.

\section{Mechanisms for Shifting the Tumor Microenvironment to Pro-Inflammatory}

The largest difference between a "cold" and a "hot" tumor microenvironment is typically considered to be related to the infiltration of anti-tumor, pro-inflammatory $\mathrm{T}$ cells in "hot" or inflamed tumors (75). Checkpoint inhibitors are emerging as the primary therapeutics used to facilitate the shift in the tumor microenvironment from "cold" to "hot" or immunologically active (76). In the case of many cancers, the checkpoint inhibiting pathways are hijacked to prevent immune cells from eliminating unhealthy or cancerous cells. The two most heavily studied checkpoint inhibitor pathways in the tumor ablation field are CTLA-4 to CD80/86, blocking the normal co-stimulatory role of CD80/86 in $\mathrm{T}$ cell activation, and $\mathrm{PD}-1$ to $\mathrm{PDL} 1 / 2$ (Figure 3). CTLA-4 is a checkpoint inhibitor most commonly associated with antigen presentation in the lymph nodes that minimizes the proliferation of T cells (76). Therapeutically, when CTLA-4 on T cells is blocked with a targeted antibody, there is an increase in overall $\mathrm{T}$ cell proliferation and an increased probability of forming a tumor-specific immune response. While anti-CTLA- 4 is used to increase T cell proliferation, PD1 inhibitors decrease $\mathrm{T}$ cell inhibition and improve function. Under normal conditions, PD-1 inhibits the activity of T cells and prevents the targeting of healthy host cells by $\mathrm{T}$ lymphocytes (76). This pathway in healthy tissues prevents overzealous immune responses that can drive autoimmunity. However, some tumor cells hijack this mechanism and increase the expression of the PD-1 ligands (PD-L1/2), which artificially reduce the activity of anti-tumor $\mathrm{T}$ cells within the tumor

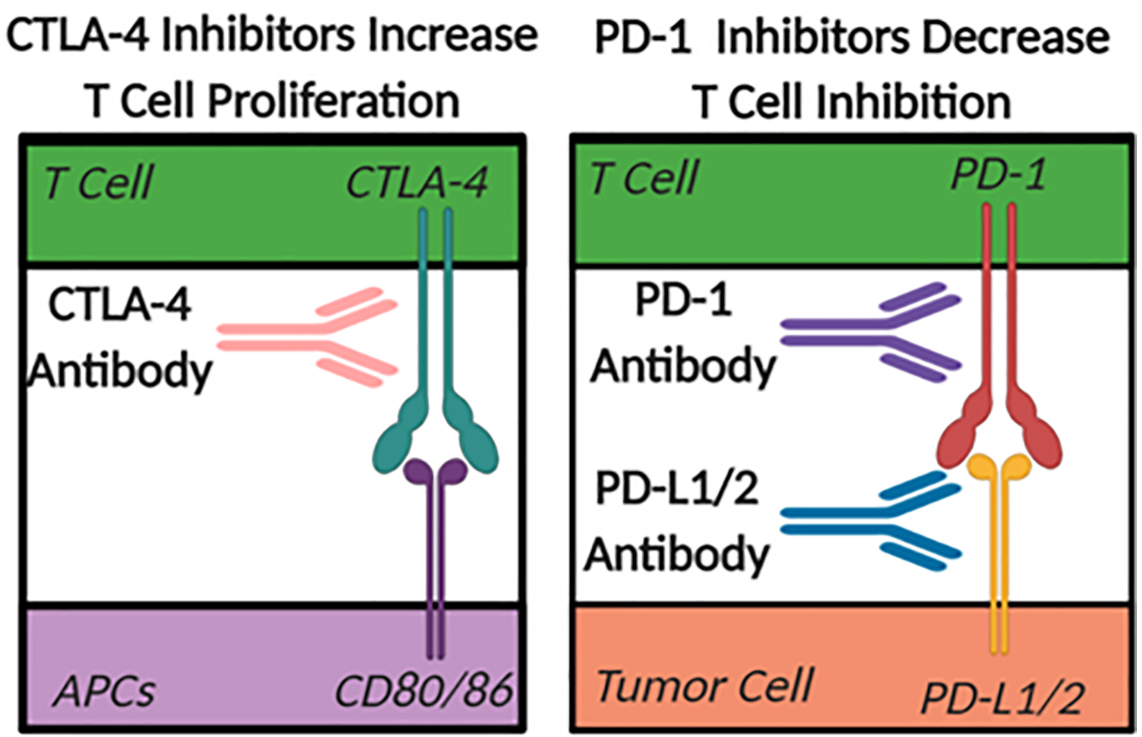

FIGURE 3 | The Role of Check Point Inhibitors. Antigen Presenting Cells (APC). 
microenvironment. The use of antibodies targeting the PD-1 pathway for cancer therapy has shown increased levels of infiltrating $\mathrm{T}$ cells in tumors that express the PD-L1/2 surface receptors. While PD-1 pathway inhibition has a more tumorspecific therapeutic effect, and typically fewer side effects compared to CTLA-4 based therapies, there is still a need to improve the implementation of checkpoint inhibition strategies. This is especially true in immunologically "cold" tumors that achieved immunosuppression without the use of PD-1 or CTLA4 pathways where these checkpoint targeted therapeutics have proven to be minimally effective. Unfortunately, even in cases where these therapies work there are often significant side effects. For example, the systemic increase in $\mathrm{T}$ cell abundance often leads to autoimmune disease-like symptoms such as fatigue, nausea, vomiting, diarrhea, arthritis, dermatitis, and myalgia (77).

Beyond the biochemical changes in the tumor microenvironment that work to subvert immune system detection and elimination, physical barriers that can have significant detrimental effects on therapeutic approaches are also present. Indeed, many tumors adopt organ-like structures and extensive fibrosis that build highly complex physical barriers $(78,79)$. Given that the checkpoint inhibitor's mechanism is to improve immune system activation and targeted killing of tumor cells, it is critical that leukocytes have physical access to the malignant cells. In an attempt aid the checkpoint inhibitor therapies, ablation modalities have been extensively studied to better understand their ability to modulate the immunological aspects of the tumor microenvironment. While debulking the tumor and reducing a patient's overall tumor burden is the primary goal of focal tumor ablation therapies, improved immune system access is proving to be an added benefit that can shift the local TME from being "cold" and immunosuppressed to more "hot" and immunostimulated.

Ablation therapies with a thermal effect have sporadically seen pro-inflammatory changes in the tumor microenvironment in the weeks and months following treatment (80-83). However, in terms of immunomodulatory success, non-thermal modalities seem to have more reproducibility, predictability, and improved pre-clinical and clinical success $(21,84-86)$. The leading hypothesis regarding differences between modalities theorizes that while both thermal and non-thermal ablation approaches kill tumor cells, non-thermal ablation modalities release higher levels of native tumor-specific antigens that have not been distorted or denatured by heat. For example, one in vitro study showed that intact neoantigens are released in significantly higher magnitudes from cells treated with cryoablation and IRE compared to thermal ablation. Not only were there more tumor antigens released, these antigens were also more potent at stimulating dendritic cell antigen presentation and cytotoxic CD8+ T cell activation (87). Activated lymphocytes, either in response to tumor-specific antigens or more generally in response to innate immune system activation driven by the increase in damage-associated molecular patterns (DAMPs) further promote the shift from a "cold" to a "hot" tumor microenvironment $(21,88,89)$. Thus, it is becoming clear that non-thermal ablation modalities are capable of inducing robust local and systemic anti-tumor responses, as either an independent or an adjuvant therapy with immunotherapeutics, to offer significant promise beyond just focal tumor debulking.

\section{HISTOTRIPSY: NON-THERMAL, NON- INVASIVE TUMOR ABLATION}

Histotripsy is a non-thermal focused ultrasound therapy that utilizes microsecond (cavitation-cloud histotripsy, $\mathrm{CCH}$ ) or millisecond (boiling histotripsy, $\mathrm{BH}$ ) pulsing regimens to generate cavitation bubble clouds that leads to precise nonthermal tumor ablation (Figure 4) (90). In addition to histotripsy, other non-thermal focused ultrasound methods that induce cavitation and do not induce substantial thermal effects are broadly referred to as mechanical HIFU (mHIFU), as opposed to conventional HIFU that typically refers to thermal ablation. For the purposes of this study, thermal HIFU will be referred to as tHIFU to delineate from mHIFU procedures, which will be the focus of this review.

In histotripsy, the rapid expansion and collapse of cavitation bubbles ablate tissues into acellular debris (91-94). This nonthermal ablation process is well-established and has resulted in important hallmarks of histotripsy, including high precision and tissue selectivity (95-97). For example, tissues with a higher Young's Modulus, such as vasculature and collecting ducts, are more resistant to damage (98-105). Unlike the vast majority of other ablation therapies, because histotripsy is non-thermal, it is not affected by the heat-sink effect, and therefore remains safe and efficacious for use near the vasculature $(106,107)$. Additional benefits of histotripsy include real-time imaging feedback with standard imaging (ultrasound, MRI, CT), highly precisemillimeter accuracy, and the ability to treat tumors of arbitrary sizes and shapes $(100,108)$. After treatment, tissues treated with histotripsy have shown more rapid dissolution of the ablated tissues compared to other ablation modalities (92, 108). For instance, $\mathrm{CCH}$ ablation of healthy rat livers showed rapid involution of treated volumes, granulation, and growth of healthy hepatocytes within 28 days with minimal scarring (92). This healing process is more rapid than what has been reported for other ablation modalities, such as RFA in humans, which has been reported as causing "thermal fixation," where the necrotic mass is still present 14 months post-treatment due to thermal denaturation of the tissue leaving it resistant to being broken down by normal pathways (109).

In mouse models, histotripsy ablation of hepatic, renal, neuroblastoma, melanoma, and pancreatic tumors has resulted in significantly increased survival $(22,110-113)$. In addition to debulking tumors, recent evidence has suggested that histotripsy has the capability to induce a systemic immune response, as evidenced by the attenuation of metastasis and an improvement in local and distant disease with combination immunotherapy $(22,114,115)$. This effect has been shown in single tumor treatments and has also shown abscopal-like decreases in contralateral tumor growth in a separate untreated tumor (22, $112,115)$. This effect was found to be modest, but statistically 


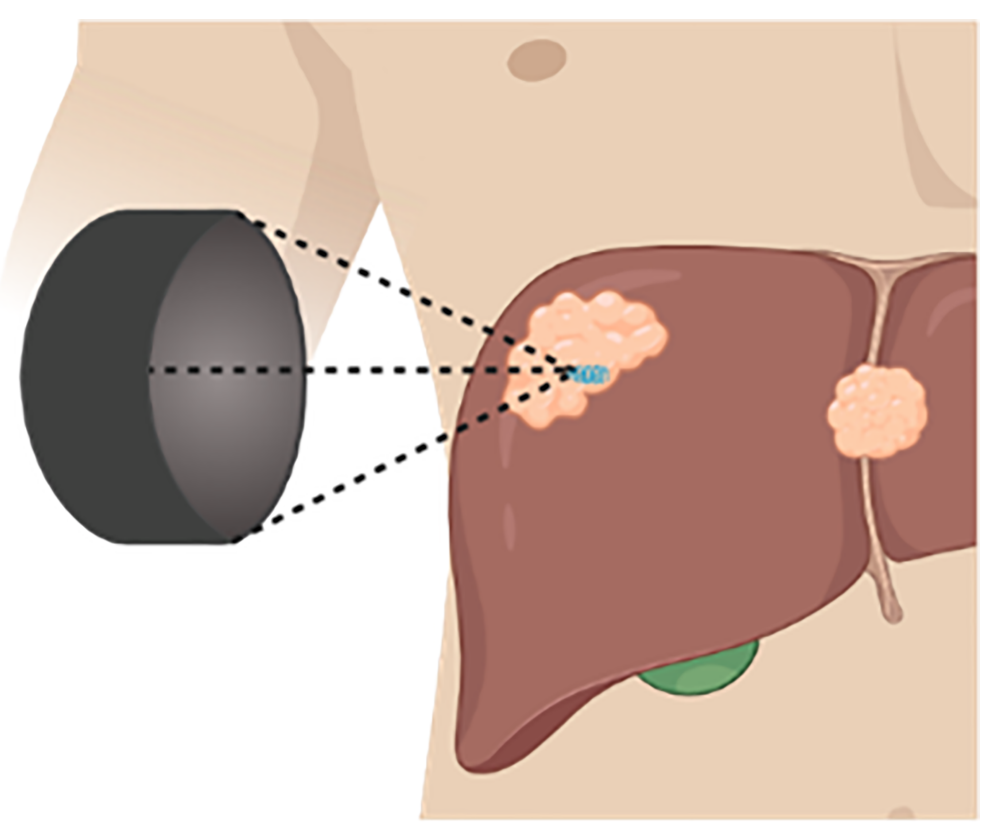

FIGURE 4 | Histotripsy Schematic. Therapeutic ultrasound transducer positioned outside of the body, focuses ultrasound waves within a targeted tissue generating a cavitation bubble cloud.

significant with histotripsy ablation compared to the insignificant trend in mice treated with irradiation and radiofrequency ablation (22). The molecular immunological effects caused by histotripsy, independently and compared to other ablation modalities, can best be summarized by the proposed mechanism shown in Figure 5 that is described in the sections below.

\section{IMMUNOLOGIC EFFECTS OF HISTOTRIPSY}

The immunological responses to histotripsy ablation through cavitation, regardless of the sub-therapy being applied, are theoretically similar due to comparable effects on targeted tissues. Cavitation breaks down tissues, ablating cells into subcellular fragments and acellular debris (116). To date, there have been no studies to suggest that $\mathrm{mHIFU}, \mathrm{BH}$, nor $\mathrm{CCH}$ have any significant differences in immunologic responses. Therefore, for this review, the immune responses to these modalities will be reviewed together.

\section{Decreases in Pro-Tumor Immune Cells}

Being able to reduce the magnitude of tumor supporting immune cells present within the tumor microenvironment is pertinent due to the correlation of these cells with poorer outcomes $(54,117,118)$. There have been multiple studies on thermal and non-thermal ablation modalities that show either the therapy directly eliminates cells that support the tumor microenvironment or the damage initiated by the ablation reprograms the leukocytes within the tumor microenvironment to shift the microenvironment to one that is more harsh towards tumor progression $(6,7)$.
In the case of histotripsy, there has been one report from Pahk et al. demonstrating the supernatant from cells treated with $\mathrm{BH}$ in vitro polarizes naïve macrophages (M0) into anti-tumor macrophages (M1) and the redifferentiation of tumor associated macrophages (M2-like phenotype) to the more inflammatory M1 phenotype (119). In this study, breast cancer cells were treated with $\mathrm{BH}$ at varying doses in vitro and the supernatant collected. Analysis found increased proinflammatory signaling molecules including TNF, which is a potent and well established M1 stimulating cytokine (120, 121). After THP-1 human monocyte cells were cultured with the $\mathrm{BH}$ supernatant they displayed physical and morphological changes consistent with polarization to M1 macrophages and showed significantly elevated gene expression consistent with M1 polarization (119). Together, the data presented in this study suggest that monocytes and macrophages near the histotripsy ablation region polarize to pro-inflammatory, anti-tumor phenotypes. Likewise, Pahk et al. continued in vitro studies showing that the $\mathrm{BH}$ supernatant can also stimulate M2 cells to repolarize to $\mathrm{M} 1$, thus leading to a decrease in $\mathrm{M} 2$ pro-tumor macrophages (Figure 5). Thus, these data further suggest that any tumor-associated macrophages remaining within the treatment zone have the potential to repolarize from an M2like state to M1, similar to the cells at the margins of treatment. If this can be recapitulated in vivo, this would help further decrease the presence of pro-tumor immune cells and could significantly alter the shift the tumor microenvironment from "cold" to "hot."

Regarding in vivo studies, it may seem safe to assume that protumor cells within the tumor microenvironment of the treatment zone would be ablated due to subcellular fractionation of 


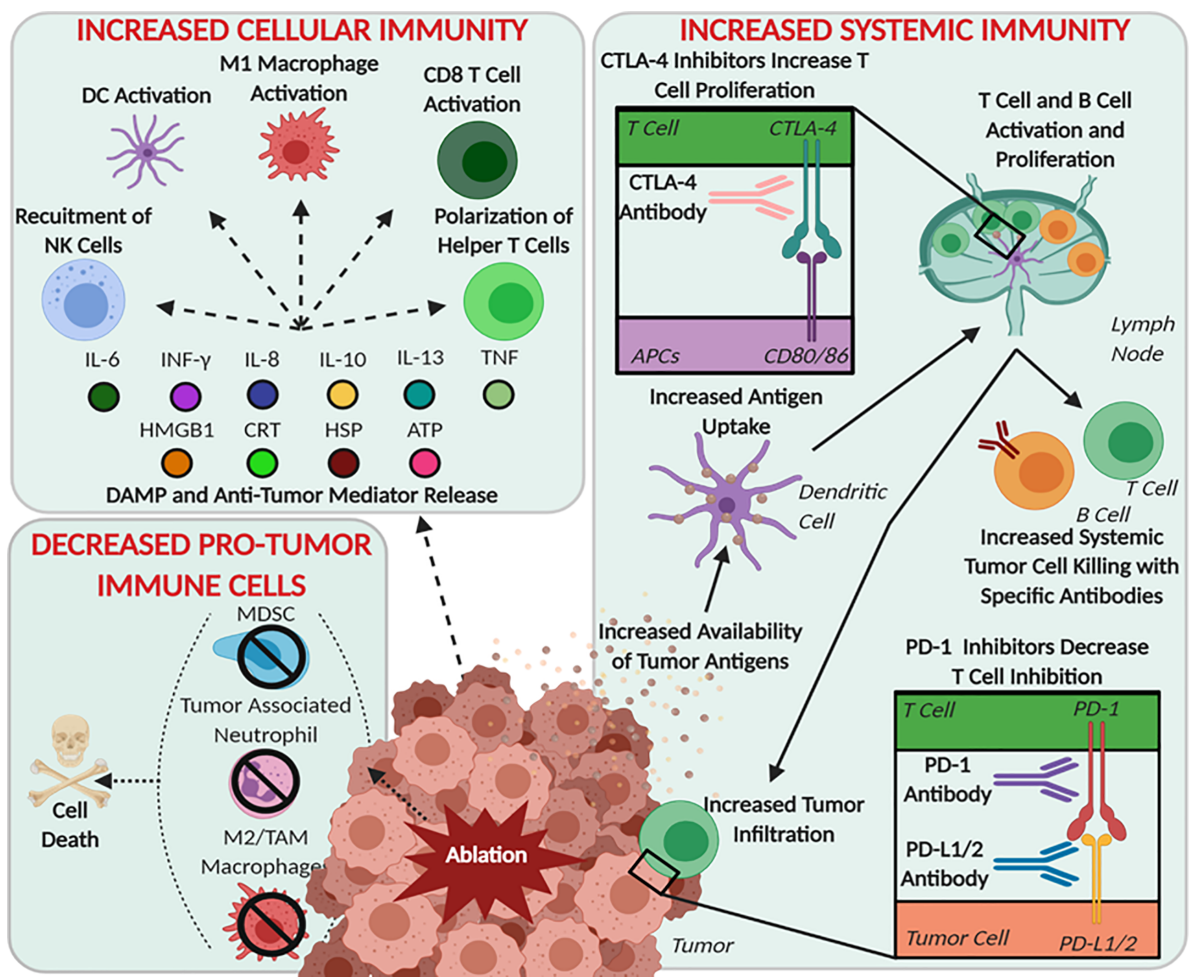

FIGURE 5 | Schematic of Histotripsy's Postulated Roles in Immune System Modulation. Immune processes, cells, and molecules that have been shown to be modulated by focused ultrasound therapies after mechanical ablation are summarized based upon immune system functions: pro-tumor immune cells, cellular immunity, and systemic immunity.

histotripsy. This specific hypothesis is not confirmed by literature, and there is still a window for any cells that survive partial ablation to proliferate and maintain the pro-tumor tumor microenvironment. However, this hypothesis is consistent with results following RFA, microwave ablation, and IRE, which can all reduce the presence of tumor supporting immune cells in the tumor microenvironment $(7,122)$. This dynamic has also been seen in human patients. For instance, studies of pancreatic adenocarcinoma patients treated with IRE found a decrease in $\mathrm{T}$ regulatory cells in peripheral blood in the days following treatment $(85,86)$. Another study of patients with primary liver tumors treated with RFA showed a strong correlation between the decrease in MDSCs and the increase in patients' survival (123). The importance of the attenuation of pro-tumor immune cells, their decrease in function, and reduction in magnitude after ablation are all generally correlated with reduced survival in human patients. Therefore, it is important for the field of histotripsy to further understand the effects of the ablation on this aspect of the immunomodulatory mechanism.

\section{Histotripsy Produces Damage Associated Molecular Patterns That Directly Increase Local Cellular Immune Responses}

Histotripsy generates subcellular fragments of targeted cells through mechanical fractionation (116). This, in turn, releases a multitude of damage associated molecular patterns (DAMPs) that have the potential to stimulate the innate immune system and significantly alter the tumor microenvironment. DAMPs are molecules that are found extracellularly following most forms of pathological cell death. These molecules are readily identified by the immune system, which in turn elicits a robust innate immune response. Several DAMPs have been observed and evaluated in the context of tumor ablation, including the endoplasmic reticulum associated protein calreticulin (CRT), the nonhistone nuclear binding protein HMGB1, the intracellular energy molecule adenosine 5'-triphosphate (ATP), and various heat shock proteins (HSP) that were originally identified as DAMPs released after exposure to elevated temperatures (124128). Once released from the injured cells or neighboring cells following damage, these molecules can stimulate a robust immune response by binding to receptors known as pattern recognition receptors. As an example, HMGB1 commonly activates the Toll-like Receptors 2 and 4, which classically signals through NF- $\kappa \mathrm{B}$ to drive multiple biological responses, including inflammation and cell death (129).

These DAMPs are emerging as key mediators of the ensuing immune response following histotripsy. Specifically, following histotripsy treatment of cancer spheroids, HMGB1, CRT, and HSP were identified in cell supernatants and correlated to levels expected from within the tumor after an in vivo ablation (119). 
Additionally, murine tumors treated with histotripsy have been found to have elevated levels of HMGB1, CRT, and HSP within the tumor and increased HMGB1 has been routinely found in serum following treatment $(22,110,130)$. DAMPs released in serum function to systemically prime the immune system and can function to recruit increased circulating leukocytes that can ultimately congregate at the site of tissue injury following ablation. Together, the local and systemic presence of DAMPs aid additional immune cell migration, shift the tumor microenvironment, and may eventually serve as effective biomarkers of ablation success.

While DAMP release has been confirmed following histotripsy, it is also critical to determine if these signals are in their naïve and unaltered structural configurations. For thermal ablation modalities, it has been well established that proteins can be denatured under high heat settings, making them less efficacious and predictable in stimulating the immune system. One study investigated the difference in the release of ATP and hsp60 between mHIFU and tHIFU (130). This study found that mHIFU is capable of releasing higher levels of both molecules after treatment, and that the DAMPs released from mHIFU were more capable of stimulating downstream immunologic changes, such as activating dendritic cells (130). The ability of mHIFU to release a higher magnitude of active DAMPs compared to tHIFU adds to the hypothesis that nonthermal ablation therapies are more immunomodulatory compared to thermal modalities.

These data point to histotripsy therapies having the ability to release molecules that can drive anti-tumor immune system activation, for example by initiating a robust innate immune response. However, the data associated with the specific DAMPs released following histotripsy is not consistent across studies. For example, a recent study of subcutaneous murine neuroblastoma treated with mHIFU showed no significant changes in HMGB1 levels (112). This could be due to a difference in tumor types. For example, neuroblastoma did not see the change in DAMPs after histotripsy that was observed in colon adenocarcinoma, renal cell carcinoma, and breast adenocarcinoma reported elsewhere (22, $110,112,130)$. Alternatively, there are potential differences in histotripsy and other mHIFU ablation modalities that are not fully understood and may have played a role. Another possibility is that differences in DAMP release between a partial ablation versus a more complete treatment are responsible for these differences. All of these variables could potentially affect the quality and quantity of DAMPs released. Likewise, the actual DAMPs generated could significantly vary depending on a number of experimental factors. Although the aforementioned DAMPs are currently the most defined following histotripsy, any DAMP could conceivably function as an activator of the innate immune system after ablation. Future investigation into the effects of other DAMPs, such as nuclear and mitochondrial DNA, reactive oxygen species, and calcium ions could prove useful for improving adjuvant therapies (131). Additional studies are needed to determine any potential difference in the quantity and quality of the release of DAMPs from the different mechanical focused ultrasound therapies.

\section{Pro-Inflammatory Cytokines and Chemokines Associated With Histotripsy Based Tumor Ablation Modalities}

Cytokines and chemokines are critical for cell-to-cell communication and immune system modulation following damage (Figure 5). These molecules stimulate the differentiation and activation of local immune cells and systemically recruit additional cells to the site of damage. One of the critical cytokines found to be significantly altered in multiple histotripsy studies is $\operatorname{IFN} \gamma(22,112,115,119)$. In an in vitro study on breast adenocarcinoma and an in vivo murine study of neuroblastoma, there was an approximately 2 -fold increase in IFN $\gamma$ after histotripsy $(112,119)$. However, in an in vivo study where Eker rat renal cell carcinoma tumors were treated with histotripsy, there was a significant decrease, $\sim 6 \mathrm{pg} / \mathrm{mL}$ down to $\sim 2 \mathrm{pg} / \mathrm{mL}$, in treated kidneys (115). This decrease was attributed to the native kidney tissues' defense mechanism to protect against acute and chronic kidney injuries (132). The importance of changes to IFN $\gamma$ is highlighted by its role in activating anti-tumor APCs and effector cells, inducing ischemia within the tumor by acting on the endothelial cells thus reducing the stability of intratumoral vasculature, and initiating tumor cell death through the activation of apoptosis and necroptosis pathways (133). However, IFN $\gamma$ is also a double-edged sword in the tumor microenvironment. In addition to its function as a potent proinflammatory mediator, IFN $\gamma$ can also up-regulate PD-L1 in some tumors as part of the IRF1 axis, resulting in immune evasion (134).

While IFN $\gamma$ is the most consistently reported inflammatory mediator across therapies and tumor types, other important cytokines have been reported as either significantly or notably upregulated in the days after histotripsy treatment including IL6, IL-2, TNF, IL-8, IL-13, and IL-10 (22, 110, 112, 119). While more research is needed to more accurately determine the state of cytokines in specific tumor types after mechanical ablation, the general picture appears positive. However, high levels of IL-6, IL-10, and IL-8 in serum have been reported to negatively correlate with survival in pancreatic cancer patients, due to their role in stimulating inflammation and adverse changes in the tumor microenvironment that spurs the growth and development of pancreatic tumors $(135,136)$. Although this could correlate to a foreboding warning against histotripsy therapies, at least for pancreatic applications, the induction of these pathways actually correlates with improved anti-tumor immune responses and increased survival in mice (22). It is certainly possible that these results simply reflect the commonly promoted differences between mice and humans. However, it is also possible that additional mechanistic insight may be necessary to better translate the findings from rodents to human patients.

In addition to the cytokines released there are changes in the serum levels of multiple growth factors as well. For example, after mHIFU ablation of neuroblastomas, both an increase in GMCSF and a decrease in VEGF were found within 24 hours of 
treatment (112). Increased levels of GM-CSF are positively correlated with increased APC cell differentiation and activation. Decreased levels of VEGF are positively correlated with better clinical outcomes, due to the rate of vascularization of tumors affecting tumor growth and immunosuppressive effects (137). The finding that histotripsy can increase GM-CSF and decrease VEGF adds to the potential for histotripsy to shift the tumor promoting immune microenvironment to one that is more proinflammatory and tumor suppressive.

\section{Histotripsy Significantly Alters Immune Cell Populations Systemically and in the Tumor Microenvironment}

With the increased release of DAMPs and anti-tumor mediators, there is a resultant change in intra-tumoral immune cell populations. In response to damage, the cells associated with innate immunity are the most rapidly recruited. For histotripsy ablation, this has been reported to include neutrophils, natural killer cells, dendritic cells, and macrophages (Figure 5). In an in vitro experiment, the supernatant of mHIFU treated murine colon adenocarcinoma cells was shown to activate macrophages (130). This macrophage activation was established through the release of TNF from the macrophages, which was significantly greater after mHIFU compared to both tHIFU and controls (130). Additionally, in vivo treatment of melanoma tumors with $\mathrm{CCH}$ showed increases in neutrophils, natural killer cells, dendritic cells, and macrophages 10 days after treatment (22). This increase in intratumoral immune cell populations indicate that the tumor microenvironment has become more immunostimulatory and these cell populations typically correlate to pro-inflammatory and anti-tumor changes in the tumor microenvironment.

Beyond innate immune cells, modulation of adaptive immune cells after ablation has also been strongly correlated with clinical success $(6,7)$. In the same melanoma $\mathrm{CCH}$ ablation study, increases in $\mathrm{T}$ helper cells and $\mathrm{B}$ cells within the treated tumors were observed 10 days post-treatment (22). In addition to the increase in these adaptive immune cell populations, the study also found a decrease in intratumoral $\mathrm{T}$ regulatory cells (22). The increase in helper T cells and B cells is a further indication that the TME has shifted to a more permissible, pro-inflammatory environment and further illustrates the improved accessibility of these cells to the tumor. In general, the influx of leukocytes appears to reflect the early induction of the innate immune system and eventually yields to cells associated with a robust adaptive immune response over the course of a few weeks following treatment. Together, these changes are predicted to have significant impacts on the local and systemic effects on metastatic lesions. However, it is critical to note that, while the increased presence of these pro-inflammatory immune cells within the residual treated tumor does hint at a stronger and more robust overall immune response, these cells must be specifically primed for tumor antigens found and either in the circulation or in secondary lymphoid organs to effectively control the overall disease burden.

\section{Improved Engagement of the Adaptive Immune System and Increases in the Systemic Anti-Tumor Immune Response}

The initiation of the systemic anti-tumor immune response requires the generation of both high quantity and high-quality tumor antigens. Previous studies with thermal ablation, cryoablation, and IRE established that the non-thermal ablation modalities appear to release antigens that are significantly better at driving predictable and effective antigen presentation (87). This ultimately results in improved systemic immune responses (87). Although an explicit study has yet to explore if histotripsy ablation negatively impacts the quality of released antigens, studies have been conducted that indirectly evaluate this mechanism. A study using the B16F10 murine melanoma cell line and a transgenic variant of the cell line revealed an increase in the immunomodulatory effects of histotripsy in the tumors that expressed an immunogenically active antigen (22). In this study, only mice that had tumors transfected with the potential antigen GP33, a known antigen from lymphocytic choriomeningitis virus, and were also treated with histotripsy, generated CD8 $+\mathrm{T}$ cells that were capable of producing IFN $\gamma$ after stimulation with IL-2 and brefeldin A. These two agents stimulate memory CD8+ T cells. Together, these data imply that transfected antigens are still viable after histotripsy treatment and are able to stimulate an adaptive immune response.

The activation and migration of dendritic cells to tumordraining lymph nodes and the spleen are further evidence of systemic immune system activation. Early studies into dendritic cells found the supernatants collected from in vitro mHIFU treatments were able to stimulate immature dendritic cells to express higher levels of CD80 and CD86, which are markers of activation, and enhanced secretion of IL-12, which are utilized by dendritic cells to activate both CD8+ cytotoxic and CD4+ helper T cells $(130,138,139)$. Additional murine studies with various tumor types further established the migration of, or the increase in, the number of dendritic cells to the tumor-draining lymph nodes and spleen $(112,140,141)$. The higher presence of this critical antigen-presenting cell indicates that a subsequent increase in lymphocyte populations is likely.

Once the APCs have been activated, antigens are presented $\mathrm{CD} 8+$ and $\mathrm{CD} 4+\mathrm{T}$ cells that proliferate and are subsequently recruited back to the treated tumor, as well as, circulate systemically to target and kill distal tumor cells expressing the targeted tumor specific antigens. As discussed earlier, the reduction of $\mathrm{T}$ regulatory cells is important for changing the tumor microenvironment and shifting the immune response from "cold" to "hot", allowing these anti-tumor CD4+ and CD8 $+\mathrm{T}$ cells access to malignant cells. Histotripsy has been shown to reduce the magnitude of $\mathrm{T}$ regulatory cells and increase the ratio of $\mathrm{CD} 8+$ to $\mathrm{T}$ regulatory cells in both the tumor-draining lymph nodes and spleens of treated mice $(22,141)$. For example, the ratio of $\mathrm{CD} 8+: \mathrm{CD} 4+$ cells in the spleen was found to increase after treating melanoma tumors with $\mathrm{CCH}$ (22). Additionally, after treating prostate tumors with mHIFU, the ratio of $\mathrm{CD} 8+: \mathrm{CD} 4+$ cells in the spleen was also found to be 
increased, and protective against subsequent tumor challenges (141). However, in treating neuroblastoma tumors with mHIFU, the ratio of $\mathrm{CD} 8+: \mathrm{CD} 4+$ cells in the spleen was found to decrease (112). This difference in the increase versus decrease of the CD8+:CD4+ ratio is not necessarily a sign that regulatory $\mathrm{T}$ cells are predominate nor is it indication of poor prognosis. This shift in ratio could indicate helper $\mathrm{T}$ cells primed to increase an immune response in response to the damage that could lead to an immunostimulatory response. Beyond regulatory $\mathrm{T}$ cells, histotripsy has been found to increase the number of CD8+ and CD4+ helper cells in the spleen $(22,112,142)$. These changes in $\mathrm{T}$ cell populations in secondary lymphoid organs after histotripsy treatment implies that similar changes occur within the tumor, and that these cells are primmed against tumor specific antigens.

While all of these studies established correlative changes in $\mathrm{T}$ cells after histotripsy treatment, it is important to determine the efficacy and tumor specificity of programmed cells. CD8+ cells isolated from the spleens of mice that had subcutaneous colon adenocarcinoma tumors 10 days post-treatment with mHIFU were co-cultured with the corresponding cell line (140). The CD8 $+\mathrm{T}$ cells were found to be more cytotoxicity against the cancer cells compared to the lymphocytes isolated from mice treated with tHIFU. In this same study, ELIspot assays showed that mHIFU generated a higher magnitude of tumor-specific CD8+ cells than tHIFU (140). In a similar study with histotripsy treated prostate tumors, CD8+ T cells harvested from the spleen were found to be tumor-specific and were subsequently activated when challenged in vitro with the tumor cells (141). Finally, in a third study, the treatment of a known antigen into a cell line before tumor engraftment allowed for the determination of CD8+ $\mathrm{T}$ cells in the tumor-draining lymph nodes that were primed against tumor-specific antigens after treatment with $\mathrm{CCH}$ (22). Together, these studies support the hypothesis that histotripsy treatment of the local tumor is effective at generating tumor specific CD8+ cytotoxic $\mathrm{T}$ cells that are found systemically in distal lymph nodes and the spleen.

The efficacy of adaptive immune system activation is shown through systemic tumor killing both through the reduction of metastasis and control of contralateral tumors. Several studies have evaluated this phenomenon. For example, in a study using immunocompetent New Zealand White rabbits with VX-2 (leporine papilloma) tumors grown within a single kidney, histotripsy treatment on day 13 did not show a change in metastasis by day 19 (143). Ultimately, the authors associated the lack of difference to the aggression of the cancer (143). Beyond this study, it should be noted that the majority of histotripsy studies have actually shown significant improvements in metastasis $(22,142)$. For example, in a murine melanoma model, tumors were grown subcutaneously, treated with histotripsy, and amputated two days after treatment (142). For most studies conducted to date, decreases in lung metastasis are correlated to systemic cancer control and suggestive of an abscopal response. However, as an alternative interpretation of the data, the decrease in metastasis could simply be an effect of the primary tumor ablation not being able to produce as many circulating tumor cells due to its reduced size.
This is a common critique of focal tumor ablation studies that report changes in metastatic burden.

Based on the current data in the field, we believe that it is likely that both interpretations are accurate; whereby, the decrease in metastasis is due to both the activation of the systemic anti-tumor immune response and the debulking of the primary tumor. Thus, it is essential that future mechanistic work in the field design experiments designed to better establish causation. For example, in addition to the prevention of additional tumor growth through reduced metastasis, there are multiple reports of $\mathrm{CD} 8+$ cytotoxic $\mathrm{T}$ cells in contralateral tumors $(22,115)$. To evaluate histotripsy in these contralateral models, mice were inoculated with bilateral subcutaneous B16GP33 melanoma tumors and after 10 days of tumor growth one of these tumors were treated. In the treated tumor, there was a sharp infiltration of CD8+ T cells that localized in the ablation margin, while over the course of a week the contralateral-untreated tumor slowly saw a perfuse increase in CD8+ T cells (22). This study establishes that in injected, identical tumors that a systemic effect can be achieved. As a de novo model of contralateral tumors, Eker rats have been deployed. Eker rats are a model with an insertion in the rat homologue of the human tuberous sclerosis gene (TSC2) that spontaneously develop multiple renal cell carcinoma tumors throughout both of their kidneys (144). In a study of boiling histotripsy, Eker rats had one approximately $0.5 \mathrm{~cm}^{3}$ tumor treated and after $48 \mathrm{hrs}$, their treated and contralateral kidneys were collected for immunohistochemistry staining for CD8+ cells. This found that if one tumor was treated then both tumor riddled kidneys would see an influx of CD8+ T cells, while sham treated rats did not see a change in either kidney (115). These contralateral tumors have been reported to have decreased growth rates after histotripsy treatment of the targeted primary tumor, and more significantly than radiation ablation or RFA (22). Using mice with B16GP33 tumors, after 10 days of tumor growth mice were either treated with histotripsy, RFA, radiation therapy, or sham-control treated. This study found that the mice that were treated with histotripsy saw a significant increase in tumor infiltrating CD8+ T cells in treated tumors compared to RFA and radiation ablation, neither of which saw any increase from sham-controls (22). This not only further strengthens the idea that histotripsy can stimulate a systemic immune response, but also supports the idea that non-thermal and non-ionizing therapies are more effective.

While there is a reduction in metastasis in these studies, the more established contralateral tumors only have their growth slowed, not inhibited nor reversed. This implies that while there is a systemic immune response against the cancer, it is not strong enough to prevent growth or be curative without combination with other therapeutic methods or additional treatments. Further, the efficacy of mHIFU treatment of generating systemic tumor-specific protection has been shown with the reduction in tumor growth rate in challenge tumors injected 6 days after treatment (140). Notable, the effects of mHIFU to minimize the growth rates of treated tumors were more effective than tHIFU, even though the debulking on the primary tumor was much greater $(83 \%$ and $43 \%$ reduction in tumor volume 
respectively) (140). This indicates that the non-thermal ablation modality stimulated a stronger immune response. However, as with the pre-existing contralateral tumors, the post-treatment injection challenge still allowed for tumor growth. Together these studies reveal that histotripsy has the power to prime the murine immune system sufficiently to reduce systemic tumor burden through control of naturally occurring metastasis, spontaneous similar-primary tumors, and secondary tumors injected as a challenge.

\section{Combination Therapy With Checkpoint-Inhibitors}

Given that many cancers are immunologically resistant to checkpoint-inhibitors due, in part, to the immunological state of the tumor microenvironment, it is critical to explore the potential of histotripsy as a strategy to improve tumor responsiveness. The increased release of DAMPs, shift in the inflammatory state of the tumor microenvironment, enhanced recruitment of immune cells within the tumor, and evidence of a systemic anti-tumor immune response all discussed above indicate that histotripsy can have local and systemic immunomodulatory effects that may be favorable for these classes of therapeutics. Based on these changes, it is reasonable to hypothesize that the shift of the tumor microenvironment to a "hot" environment should increase the effects of checkpoint inhibitors allowing for a potentially stronger anti-tumor immune response. The efficacy of anti-CTLA-4, anti-PD-1, and anti-PD-L1 therapies is correlative to the levels of expression of CTLA-4, CD80/86, PD-1, and PD-L1 in the patients' tumor cells, APCs, and T cells (Figure 3). Therefore, before studying the effects of specific checkpoint inhibitors, it is critical to determine if there is supporting data to suggest that histotripsy may have a synergistic effect (76). For example, anti-PD-L1 targeted therapies are not typically used in cases where tumors do not express high levels of PD-L1. However, this is not always a clear decision, especially for checkpoint inhibitors that can be induced or up-regulated following treatment. Take for instance the expression of CD80/86 on dendritic cells, which was found to increase in vitro when stimulated by mHIFU supernatant, indicating that the lysate released from cells after histotripsy treatment can stimulate an upregulation of a checkpoint inhibitor (130). In vivo, CD86 was significantly increased on dendritic cells found in the spleen, and CD80 was increased significantly on dendritic cells in both spleen and tumor draining lymph nodes (141). It has also been shown that there is increase in PD-L1 in tumors 72 hours after treatment with mHIFU (112). As mentioned above, this could be due to the downstream effects of IFN $\gamma$ production following treatment. While this is typically detrimental to the systemic immune response, these in vivo upregulations of checkpoint molecules after histotripsy treatment indicates that there may be an increased window of opportunity to utilize checkpoint inhibitors for synergistic therapy to potentially improve anti-tumor immunity.

With the multiple studies suggesting that histotripsy ablation can increase the expression of both CTLA-4 and PD-1 pathway receptors, it is reasonable to hypothesize that histotripsy in combination with checkpoint inhibitor therapies should have a synergistic effect. This has been explored in a pair of recent studies. In one study, individual CTLA-4 antibody therapy and $\mathrm{CCH}$ ablation decreased the rate of contralateral tumor growth, but there was an even more significant decrease with combined therapy in both melanoma and hepatocellular carcinoma (22). For this study, one group of mice bearing subcutaneous B16GP33 melanoma tumors were administered two doses of CTLA-4 monoclonal antibody prior to histotripsy and an additional treatment afterwards. Another group of mice bearing subcutaneous Hepa1-6 hepatocellular carcinoma tumors were given three doses of CTLA-4 monoclonal antibody prior and one post histotripsy treatment. For both of these tumor studies, melanoma and hepatocellular carcinoma, the combination of CTLA-4 and histotripsy yielded significantly reduced tumor volume compared to either treatment individually (22). In a second study, three doses of both CTLA-4 and PD-L1 antibodies in the days following mHIFU treatment of subcutaneous neuroblastoma tumors significantly increased the survival of animals compared to other therapeutic combinations (112). This study went further and performed a similar experiment, but instead of only looking at the effects of a single mHIFU treated tumor there was a second tumor grown contralaterally. In this report, after unilateral treatment with mHIFU and systemic CTLA-4 and PD-L1 therapy there was complete remission of both tumors, an effect that was not seen with any other therapy combination (112). Together, these studies demonstrate the improved therapeutic effects of the combined therapy over either histotripsy or checkpoint inhibitors independently in the murine models.

\section{CONCLUSION AND FUTURE OUTLOOK}

As histotripsy therapies have been developed over the past decade, the knowledge about the immune response has started to develop a more complete picture about the hypothetical mechanisms of action from DAMP and anti-tumor mediator release, to changes in local cellular immune populations, development of a systemic immune response, and therapeutic synergism with the inclusion of checkpoint inhibitor therapies (Figure 5). In sum, these studies suggest that within murine models there is a reproducible and perhaps even tunable immune effect generated by histotripsy modalities that is consistent across multiple tumor types. However, while these data are certainly exciting, the number of studies in this field are still quite limited and most are based on murine models with inconsistent tumor ablation quality. Looking towards the future, there needs to be an effort to compare the various histotripsy treatments and doses, as well as other mHIFU methods, in order to better understand the relationship between the extent of ablation in stimulating an immune response. Likewise, as these therapies begin to be utilized in human trials, it will be crucial to translate these findings into actionable results relevant to human patients. Additional basic studies and preclinical animal trials are also still needed to develop missing mechanistic insight and more translationally relevant studies are needed to ensure these findings occur outside of the typical model organisms. Despite 
these limitations, the benefits of histotripsy over other thermal ablation modalities in pre-clinical work suggest the potential of this focal tumor ablation therapy to induce a systemic anti-tumor immune response and therefore supports the hypothesis that histotripsy has the potential to positively impact the clinical outcomes for cancer patients.

\section{AUTHOR CONTRIBUTIONS}

All authors contributed to the article and approved the submitted version.

\section{REFERENCES}

1. Befeler AS, Di Bisceglie AM. Hepatocellular Carcinoma: Diagnosis and Treatment. Gastroenterology (2002) 122(6):1609-19. doi: 10.1053/ gast.2002.33411

2. Chiorean EG, Coveler AL. Pancreatic Cancer: Optimizing Treatment Options, New, and Emerging Targeted Therapies. Drug design Dev Ther (2015) 9:3529-45. doi: 10.2147/DDDT.S60328

3. Armengol C, Sarrias MR, Sala M. Hepatocellular Carcinoma: Present and Future. Medicina Clinica (English Edition) (2018) 150(10):390-7. doi: 10.1016/j.medcle.2017.08.024

4. Hsieh JJ, Purdue MP, Signoretti S, Swanton C, Albiges L, Schmidinger M, et al. Renal Cell Carcinoma. Nat Rev Dis Primers (2017) 3:17009. doi: 10.1038/nrdp.2017.9

5. Adam R, Kitano Y, Abdelrafee A, Allard M-A, Baba H. Debulking Surgery for Colorectal Liver Metastases: Foolish or Chance? Surg Oncol (2020) 33:266-9. doi: 10.1016/j.suronc.2020.02.008

6. Kepp O, Marabelle A, Zitvogel L, Kroemer G. Oncolysis Without VirusesInducing Systemic Anticancer Immune Responses With Local Therapies. Nat Rev Clin Oncol (2019) 17:1-16. doi: 10.1038/s41571-019-0272-7

7. Keisari Y. Tumor Abolition and Antitumor Immunostimulation by PhysicoChemical Tumor Ablation. Front Biosci (Landmark Ed) (2017) 22:310-47. doi: $10.2741 / 4487$

8. Capps J, Smith J. Experiments on the Leukolytic Action of the Blood Serum of Cases of Leukaemia Treated With X-Ray and the Injection of Human Leukolytic Serum in a Case of Leukaemia. J Exp Med (1907) 9(1):51-63. doi: 10.1084/jem.9.1.51

9. Senn N. Case of Splenomedullary Leukaemia Successfully Treated by the Use of the Roentgen. Med Rec (1903) 64:281-2.

10. Gunz F. Bone Marrow Changes in Patients With Chronic Leukemia Treated by Splenic X-Irradiation: Preliminary Report. Blood (1953) 8(8):687-92. doi: 10.1182/blood.V8.8.687.687

11. Hotchkiss DJ, Block MH. Effect of Splenic Irradiation on Systemic Hematopoiesis. Arch Internal Med (1962) 109(6):695-711. doi: 10.1001/ archinte.1962.03620180057007

12. Nobler MP. The Abscopal Effect in Malignant Lymphoma and Its Relationship to Lymphocyte Circulation. Radiology (1969) 93(2):410-2. doi: 10.1148/93.2.410

13. Antoniades J, Brady LW, Lightfoot DA. Lymphangiographic Demonstration of the Abscopal Effect in Patients With Malignant Lymphomas. Int $J$ Radiat Oncol Biol Phys (1977) 2(1-2):141-7. doi: 10.1016/0360-3016(77)90020-7

14. Sanchez-Ortiz RF, Tannir N, Ahrar K, Wood CG. Spontaneous Regression of Pulmonary Metastases From Renal Cell Carcinoma After Radio Frequency Ablation of Primary Tumor: in Situ Tumor Vaccine? J Urol (2003) 170(1):178-9. doi: 10.1097/01.ju.0000070823.38336.7b

15. Yang R, Reilly CR, Rescorla FJ, Sanghvi NT, Fry FJ, Franklin TD Jr, et al. Effects of High-Intensity Focused Ultrasound in the Treatment of Experimental Neuroblastoma. J Pediatr Surg (1992) 27(2):246-51. doi: 10.1016/0022-3468(92)90321-W

16. Kaminski JM, Shinohara E, Summers JB, Niermann KJ, Morimoto A, Brousal J. The Controversial Abscopal Effect. Cancer Treat Rev (2005) 31 (3):159-72. doi: 10.1016/j.ctrv.2005.03.004

\section{FUNDING}

This work was supported by the Virginia Maryland College of Veterinary Medicine; The Virginia Tech Institute for Critical Technology and Applied Sciences Center for Engineered Health; the Focused Ultrasound Foundation; and The National Institutes of Health.

\section{ACKNOWLEDGMENTS}

All figures were created using Biorender.com.

17. Demaria S, Ng B, Devitt ML, Babb JS, Kawashima N, Liebes L, et al. Ionizing Radiation Inhibition of Distant Untreated Tumors (Abscopal Effect) Is Immune Mediated. Int J Radiat Oncol Biol Phys (2004) 58(3):862-70. doi: 10.1016/j.ijrobp.2003.09.012

18. Wang D, Zhang X, Gao Y, Cui X, Yang Y, Mao W, et al. Research Progress and Existing Problems for Abscopal Effect. Cancer Manag Res (2020) 12:6695-706. doi: 10.2147/CMAR.S245426

19. Kondo S, Okusaka T, Ueno H, Ikeda M, Morizane C. Spontaneous Regression of Hepatocellular Carcinoma. Int J Clin Oncol (2006) 11 (5):407-11. doi: 10.1007/s10147-006-0591-4

20. Kim H, Park BK, Kim CK. Spontaneous Regression of Pulmonary and Adrenal Metastases Following Percutaneous Radiofrequency Ablation of a Recurrent Renal Cell Carcinoma. Korean J Radiol (2008) 9(5):470. doi: 10.3348/kjr.2008.9.5.470

21. Ringel-Scaia VM, Beitel-White N, Lorenzo MF, Brock RM, Huie KE, Coutermarsh-Ott S, et al. High-Frequency Irreversible Electroporation Is an Effective Tumor Ablation Strategy That Induces Immunologic Cell Death and Promotes Systemic Anti-Tumor Immunity. EBioMedicine (2019) 44:112-25. doi: 10.1016/j.ebiom.2019.05.036

22. Qu S, Worlikar T, Felsted AE, Ganguly A, Beems MV, Hubbard R, et al. Non-Thermal Histotripsy Tumor Ablation Promotes Abscopal Immune Responses That Enhance Cancer Immunotherapy. I ImmunoTherapy Cancer (2020) 8(1):470-2. doi: 10.1136/jitc-2019-000200

23. Scheffer HJ, Stam AG, Geboers B, Vroomen LG, Ruarus A, de Bruijn B, et al. Irreversible Electroporation of Locally Advanced Pancreatic Cancer Transiently Alleviates Immune Suppression and Creates a Window for Antitumor T Cell Activation. Oncoimmunology (2019) 8(11):1652532. doi: 10.1080/2162402X.2019.1652532

24. Lu P, Zhu X-Q, Xu Z-L, Zhou Q, Zhang J, Wu F. Increased Infiltration of Activated Tumor-Infiltrating Lymphocytes After High Intensity Focused Ultrasound Ablation of Human Breast Cancer. Surgery (2009) 145(3):28693. doi: 10.1016/j.surg.2008.10.010

25. Dromi SA, Walsh MP, Herby S, Traughber B, Xie J, Sharma KV, et al. Radiofrequency Ablation Induces Antigen-Presenting Cell Infiltration and Amplification of Weak Tumor-Induced Immunity. Radiology (2009) 251 (1):58-66. doi: 10.1148/radiol.2511072175

26. den Brok MH, Sutmuller RP, van der Voort R, Bennink EJ, Figdor CG, Ruers TJ, et al. In Situ Tumor Ablation Creates an Antigen Source for the Generation of Antitumor Immunity. Cancer Res (2004) 64(11):4024-9. doi: 10.1158/0008-5472.CAN-03-3949

27. Ito F, Vardam TD, Appenheimer MM, Eng KH, Gollnick SO, Muhitch JB, et al. In Situ Thermal Ablation Augments Antitumor Efficacy of Adoptive T Cell Therapy. Int J Hyperthermia (2019) 36(sup1):22-36. doi: 10.1080/ 02656736.2019.1653500

28. Wu F, Wang Z-B, Lu P, Xu Z-L, Chen W-Z, Zhu H, et al. Activated AntiTumor Immunity in Cancer Patients After High Intensity Focused Ultrasound Ablation. Ultrasound Med Biol (2004) 30(9):1217-22. doi: 10.1016/j.ultrasmedbio.2004.08.003

29. Widenmeyer M, Shebzukhov Y, Haen SP, Schmidt D, Clasen S, Boss A, et al. Analysis of Tumor Antigen-Specific T Cells and Antibodies in Cancer Patients Treated With Radiofrequency Ablation. Int J Cancer (2011) 128 (11):2653-62. doi: 10.1002/ijc.25601 
30. Wissniowski TT, Hänsler J, Neureiter D, Frieser M, Schaber S, Esslinger B, et al. Activation of Tumor-Specific T Lymphocytes by Radio-Frequency Ablation of the VX2 Hepatoma in Rabbits. Cancer Res (2003) 63(19):6496-500.

31. Leuchte K, Staib E, Thelen M, Gödel P, Lechner A, Zentis P, et al. Microwave Ablation Enhances Tumor-Specific Immune Response in Patients With Hepatocellular Carcinoma. Cancer Immunology Immunotherapy (2020) 70:1-15. doi: 10.1007/s00262-020-02734-1

32. Dan J, Zhang Y, Peng Z, Huang J, Gao H, Xu L, et al. Postoperative Neutrophil-to-Lymphocyte Ratio Change Predicts Survival of Patients With Small Hepatocellular Carcinoma Undergoing Radiofrequency Ablation. PloS One (2013) 8(3):e58184. doi: 10.1371/journal.pone.0058184

33. Ahmed M, Kumar G, Moussa M, Rozenblum N, Goldberg S. Radiofrequency (RF) Ablation of Normal Liver Increases Tumor Growth of Remote Subcutaneous Tumors in an Animal Tumor Model. J Vasc Interventional Radiol (2013) 24(4):S44. doi: 10.1016/j.jvir.2013.01.098

34. Ohno T, Kawano K, Yokoyama H, Tahara K, Sasaki A, Aramaki M, et al. Microwave Coagulation Therapy Accelerates Growth of Cancer in Rat Liver. $J$ hepatology (2002) 36(6):774-9. doi: 10.1016/S0168-8278(02)00058-2

35. Ahmed M, Kumar G, Gourevitch S, Levchenko T, Galun E, Torchilin V, et al. Radiofrequency Ablation (RFA)-Induced Systemic Tumor Growth can be Reduced by Suppression of Resultant Heat Shock Proteins. Int J Hyperthermia (2018) 34(7):934-42. doi: 10.1080/02656736.2018.1462535

36. Ahmed M, Kumar G, Moussa M, Wang Y, Rozenblum N, Galun E, et al. Hepatic Radiofrequency Ablation-Induced Stimulation of Distant Tumor Growth Is Suppressed by c-Met Inhibition. Radiology (2016) 279(1):103-17. doi: 10.1148/radiol.2015150080

37. Velez E, Goldberg SN, Kumar G, Wang Y, Gourevitch S, Sosna J, et al. Hepatic Thermal Ablation: Effect of Device and Heating Parameters on Local Tissue Reactions and Distant Tumor Growth. Radiology (2016) 281 (3):782-92. doi: 10.1148/radiol.2016152241

38. Distelmaier M, Barabasch A, Heil P, Kraemer NA, Isfort P, Keil S, et al. Midterm Safety and Efficacy of Irreversible Electroporation of Malignant Liver Tumors Located Close to Major Portal or Hepatic Veins. Radiology (2017) 285(3):1023-31. doi: 10.1148/radiol.2017161561

39. Ostuni R, Kratochvill F, Murray PJ, Natoli G. Macrophages and Cancer: From Mechanisms to Therapeutic Implications. Trends Immunol (2015) 36 (4):229-39. doi: 10.1016/j.it.2015.02.004

40. Fu L-Q, Du W-L, Cai M-H, Yao J-Y, Zhao Y-Y, Mou X-Z. The Roles of Tumor-Associated Macrophages in Tumor Angiogenesis and Metastasis. Cell Immunol (2020) 104119. doi: 10.1016/j.cellimm.2020.104119

41. Wu K, Lin K, Li X, Yuan X, Xu P, Ni P, et al. Redefining Tumor-Associated Macrophage Subpopulations and Functions in the Tumor Microenvironment. Front Immunol (2020) 11:1731. doi: 10.3389/fimmu. 2020.01731

42. Zhou K, Cheng T, Zhan J, Peng X, Zhang Y, Wen J, et al. Targeting TumorAssociated Macrophages in the Tumor Microenvironment. Oncol Lett (2020) 20(5):234. doi: 10.3892/ol.2020.12097

43. Youn JI, Gabrilovich DI. The Biology of Myeloid-Derived Suppressor Cells: The Blessing and the Curse of Morphological and Functional Heterogeneity. Eur J Immunol (2010) 40(11):2969-75. doi: 10.1002/eji.201040895

44. Ko JS, Zea AH, Rini BI, Ireland JL, Elson P, Cohen P, et al. Sunitinib Mediates Reversal of Myeloid-Derived Suppressor Cell Accumulation in Renal Cell Carcinoma Patients. Clin Cancer Res an Off J Am Assoc Cancer Res (2009) 15(6):2148-57. doi: 10.1158/1078-0432.CCR-08-1332

45. Rodriguez PC, Ernstoff MS, Hernandez C, Atkins M, Zabaleta J, Sierra R, et al. Arginase I-Producing Myeloid-Derived Suppressor Cells in Renal Cell Carcinoma Are a Subpopulation of Activated Granulocytes. Cancer Res (2009) 69(4):1553-60. doi: 10.1158/0008-5472.CAN-08-1921

46. Fridlender ZG, Sun J, Kim S, Kapoor V, Cheng G, Ling L, et al. Polarization of Tumor-Associated Neutrophil Phenotype by TGF- $\beta$ :"N1" Versus "N2" Tan. Cancer Cell (2009) 16(3):183-94. doi: 10.1016/j.ccr.2009.06.017

47. Wu L, Zhang XH. Tumor-Associated Neutrophils and MacrophagesHeterogenous But Not Chaotic. Front Immunol (2020) 11:553967. doi: 10.3389/fimmu. 2020.553967

48. Hajizadeh F, Maleki LA, Alexander M, Mikhailova MV, Masjedi A, Ahmadpour M, et al. Tumor-Associated Neutrophils as New Players in Immunosuppressive Process of the Tumor Microenvironment in Breast Cancer. Life Sci (2020) 118699. doi: 10.1016/j.lfs.2020.118699
49. Summers C, Rankin SM, Condliffe AM, Singh N, Peters AM, Chilvers ER. Neutrophil Kinetics in Health and Disease. Trends Immunol (2010) 31 (8):318-24. doi: 10.1016/j.it.2010.05.006

50. van Raam BJ, Drewniak A, Groenewold V, van den Berg TK, Kuijpers TW. Granulocyte Colony-Stimulating Factor Delays Neutrophil Apoptosis by Inhibition of Calpains Upstream of Caspase-3. Blood J Am Soc Hematol (2008) 112(5):2046-54. doi: 10.1182/blood-2008-04-149575

51. Wislez M, Fleury-Feith J, Rabbe N, Moreau J, Cesari D, Milleron B, et al. Tumor-Derived Granulocyte-Macrophage Colony-Stimulating Factor and Granulocyte Colony-Stimulating Factor Prolong the Survival of Neutrophils Infiltrating Bronchoalveolar Subtype Pulmonary Adenocarcinoma. Am J Pathol (2001) 159(4):1423-33. doi: 10.1016/S0002-9440(10)62529-1

52. Trellakis S, Farjah H, Bruderek K, Dumitru C, Hoffmann T, Lang S, et al. Peripheral Blood Neutrophil Granulocytes From Patients With Head and Neck Squamous Cell Carcinoma Functionally Differ From Their Counterparts in Healthy Donors. Int J immunopathology Pharmacol (2011) 24(3):683-93. doi: 10.1177/039463201102400314

53. Najafi M, Hashemi Goradel N, Farhood B, Salehi E, Nashtaei MS, Khanlarkhani N, et al. Macrophage Polarity in Cancer: A Review. J Cell Biochem (2019) 120(3):2756-65. doi: 10.1002/jcb.27646

54. Wu L, Saxena S, Awaji M, Singh RK. Tumor-Associated Neutrophils in Cancer: Going Pro. Cancers (2019) 11(4):564. doi: 10.3390/cancers11040564

55. Tesi R. MDSC; the Most Important Cell You Have Never Heard of. Trends Pharmacol Sci (2019) 40(1):4-7. doi: 10.1016/j.tips.2018.10.008

56. Pang L, Han S, Jiao Y, Jiang S, He X, Li P. Bu Fei Decoction Attenuates the Tumor Associated Macrophage Stimulated Proliferation, Migration, Invasion and Immunosuppression of Non-Small Cell Lung Cancer, Partially Via IL-10 and PD-L1 Regulation. Int J Oncol (2017) 51(1):25-38. doi: $10.3892 /$ ijo. 2017.4014

57. Arlauckas SP, Garren SB, Garris CS, Kohler RH, Oh J, Pittet MJ, et al. Arg1 Expression Defines Immunosuppressive Subsets of Tumor-Associated Macrophages. Theranostics (2018) 8(21):5842. doi: 10.7150/thno.26888

58. Lang R, Patel D, Morris JJ, Rutschman RL, Murray PJ. Shaping Gene Expression in Activated and Resting Primary Macrophages by IL-10. J Immunol (2002) 169(5):2253-63. doi: 10.4049/jimmunol.169.5.2253

59. Wan S, Zhao E, Kryczek I, Vatan L, Sadovskaya A, Ludema G, et al. TumorAssociated Macrophages Produce Interleukin 6 and Signal Via STAT3 to Promote Expansion of Human Hepatocellular Carcinoma Stem Cells. Gastroenterology (2014) 147(6):1393-404. doi: 10.1053/j.gastro.2014.08.039

60. Bolat F, Kayaselcuk F, Nursal T, Yagmurdur M, Bal N, Demirhan B. Microvessel Density, VEGF Expression, and Tumor-Associated Macrophages in Breast Tumors: Correlations With Prognostic Parameters. Vascular (2006) 14:15.

61. Hu JM, Liu K, Liu JH, Jiang XL, Wang XL, Yang L, et al. The Increased Number of Tumor-Associated Macrophage Is Associated With Overexpression of VEGF-C, Plays an Important Role in Kazakh ESCC Invasion and Metastasis. Exp Mol Pathol (2017) 102(1):15-21. doi: 10.1016/ j.yexmp.2016.12.001

62. Gao X, Sui H, Zhao S, Gao X, Su Y, Qu P. Immunotherapy Targeting Myeloid-Derived Suppressor Cells (Mdscs) in Tumor Microenvironment. Front Immunol (2020) 11:585214. doi: 10.3389/fimmu.2020.585214

63. Mabuchi S, Sasano T, Komura N. Targeting Myeloid-Derived Suppressor Cells in Ovarian Cancer. Cells. (2021) 10(2). doi: 10.3390/cells10020329

64. Zhou H, Jiang M, Yuan H, Ni W, Tai G. Dual Roles of Myeloid-Derived Suppressor Cells Induced by Toll-Like Receptor Signaling in Cancer. Oncol Lett (2021) 21(2):149. doi: 10.3892/ol.2020.12410

65. Tanaka A, Sakaguchi S. Targeting Treg Cells in Cancer Immunotherapy. Eur J Immunol (2019) 49(8):1140-6. doi: 10.1002/eji.201847659

66. Curiel TJ, Coukos G, Zou L, Alvarez X, Cheng P, Mottram P, et al. Specific Recruitment of Regulatory T Cells in Ovarian Carcinoma Fosters Immune Privilege and Predicts Reduced Survival. Nat Med (2004) 10(9):942-9. doi: $10.1038 / \mathrm{nm} 1093$

67. Vizio B, Novarino A, Giacobino A, Cristiano C, Prati A, Ciuffreda L, et al. Potential Plasticity of T Regulatory Cells in Pancreatic Carcinoma in Relation to Disease Progression and Outcome. Exp Ther Med (2012) 4 (1):70-8. doi: 10.3892/etm.2012.553

68. Kindlund B, Sjöling Å, Yakkala C, Adamsson J, Janzon A, Hansson LE, et al. CD4(+) Regulatory T Cells in Gastric Cancer Mucosa Are Proliferating and 
Express High Levels of IL-10 But Little TGF- $\beta$. Gastric Cancer (2017) 20 (1):116-25. doi: 10.1007/s10120-015-0591-z

69. Levings MK, Bacchetta R, Schulz U, Roncarolo MG. The Role of IL-10 and TGF- $\beta$ in the Differentiation and Effector Function of T Regulatory Cells. Int Arch Allergy Immunol (2002) 129(4):263-76. doi: 10.1159/000067596

70. Cao X, Cai SF, Fehniger TA, Song J, Collins LI, Piwnica-Worms DR, et al. Granzyme B and Perforin Are Important for Regulatory T Cell-Mediated Suppression of Tumor Clearance. Immunity (2007) 27(4):635-46. doi: 10.1016/j.immuni.2007.08.014

71. Kalathil S, Lugade AA, Miller A, Iyer R, Thanavala Y. Higher Frequencies of GARP + Ctla-4+ Foxp3+ T Regulatory Cells and Myeloid-Derived Suppressor Cells in Hepatocellular Carcinoma Patients Are Associated With Impaired T-Cell Functionality. Cancer Res (2013) 73(8):2435-44. doi: 10.1158/0008-5472.CAN-12-3381

72. Liu S, Zhang C, Wang B, Zhang H, Qin G, Li C, et al. Regulatory T Cells Promote Glioma Cell Stemness Through TGF- $\beta$-Nf-kb-IL6-STAT3 Signaling. Cancer Immunol Immunother (2021) 113. doi: 10.1007/s00262021-02872-0

73. Salama P, Phillips M, Grieu F, Morris M, Zeps N, Joseph D, et al. TumorInfiltrating FOXP3+ T Regulatory Cells Show Strong Prognostic Significance in Colorectal Cancer. J Clin Oncol (2009) 27(2):186-92. doi: 10.1200/ JCO.2008.18.7229

74. Ladoire S, Martin F, Ghiringhelli F. Prognostic Role of FOXP3+ Regulatory T Cells Infiltrating Human Carcinomas: The Paradox of Colorectal Cancer. Cancer Immunology Immunotherapy (2011) 60(7):909-18. doi: 10.1007/ s00262-011-1046-y

75. Gajewski TF. The Next Hurdle in Cancer Immunotherapy: Overcoming the Non-T-Cell-Inflamed Tumor Microenvironment. Semin Oncol (2015) 42 (4):663-71. doi: 10.1053/j.seminoncol.2015.05.011

76. Wilky BA. Immune Checkpoint Inhibitors: The Linchpins of Modern Immunotherapy. Immunological Rev (2019) 290(1):6-23. doi: 10.1111/ imr. 12766

77. Arnaud-Coffin P, Maillet D, Gan HK, Stelmes JJ, You B, Dalle S, et al. A Systematic Review of Adverse Events in Randomized Trials Assessing Immune Checkpoint Inhibitors. Int J Cancer (2019) 145(3):639-48. doi: $10.1002 / \mathrm{ijc} .32132$

78. Plate J. Clinical Trials of Vaccines for Immunotherapy in Pancreatic Cancer. Expert Rev Vaccines (2011) 10(6):825-36. doi: 10.1586/erv.11.77

79. Jiang H, Hegde S, DeNardo DG. Tumor-Associated Fibrosis as a Regulator of Tumor Immunity and Response to Immunotherapy. Cancer Immunology Immunotherapy (2017) 66(8):1037-48. doi: 10.1007/s00262-017-2003-1

80. Yin J, Dong J, Gao W, Wang Y. A Case Report of Remarkable Response to Association of Radiofrequency Ablation With Subsequent Atezolizumab in Stage IV Nonsmall Cell Lung Cancer. Medicine. (2018) 97(44). doi: 10.1097/ MD.0000000000013112

81. Minami Y, Nishida N, Kudo M. Radiofrequency Ablation of Liver Metastasis: Potential Impact on Immune Checkpoint Inhibitor Therapy. Eur Radiol (2019) 29(9):5045-51. doi: 10.1007/s00330-019-06189-6

82. Xie C, Duffy AG, Mabry-Hrones D, Wood B, Levy E, Krishnasamy V, et al. Tremelimumab in Combination With Microwave Ablation in Patients With Refractory Biliary Tract Cancer. Hepatology (2019) 69(5):2048-60. doi: 10.1002/hep.30482

83. Saccomandi P, Lapergola A, Longo F, Schena E, Quero G. Thermal Ablation of Pancreatic Cancer: A Systematic Literature Review of Clinical Practice and Pre-Clinical Studies. Int J Hyperthermia (2018) 35(1):398-418. doi: 10.1080/02656736.2018.1506165

84. Bastianpillai C, Petrides N, Shah T, Guillaumier S, Ahmed HU, Arya M. Harnessing the Immunomodulatory Effect of Thermal and non-Thermal Ablative Therapies for Cancer Treatment. Tumor Biol (2015) 36(12):913746. doi: 10.1007/s13277-015-4126-3

85. Beitel-White N, Martin RCG, Li Y, Brock RM, Allen IC, Davalos RV. RealTime Prediction of Patient Immune Cell Modulation During Irreversible Electroporation Therapy. Sci Rep (2019) 9(1):17739. doi: 10.1038/s41598019-53974-w

86. Pandit H, Hong YK, Li Y, Rostas J, Pulliam Z, Li SP, et al. Evaluating the Regulatory Immunomodulation Effect of Irreversible Electroporation (IRE) in Pancreatic Adenocarcinoma. Ann Surg Oncol (2019) 26(3):800-6. doi: 10.1245/s10434-018-07144-3
87. Shao Q, O’Flanagan S, Lam T, Roy P, Pelaez F, Burbach BJ, et al. Engineering $\mathrm{T}$ Cell Response to Cancer Antigens by Choice of Focal Therapeutic Conditions. Int J Hyperthermia (2019) 36(1):130-8. doi: 10.1080/02656 736.2018 .1539253

88. Domingo-Musibay E, Heun JM, Nevala WK, Callstrom M, Atwell T, Galanis E, et al. Endogenous Heat-Shock Protein Induction With or Without Radiofrequency Ablation or Cryoablation in Patients With Stage Iv Melanoma. Oncologist (2017) 22(9):1026-e93. doi: 10.1634/the oncologist.2017-0060

89. Chandra D, Jahangir A, Cornelis F, Rombauts K, Meheus L, Jorcyk CL, et al. Cryoablation and Meriva Have Strong Therapeutic Effect on Triple-Negative Breast Cancer. Oncoimmunology (2016) 5(1):e1049802. doi: 10.1080/ 2162402X.2015.1049802

90. Bader KB, Vlaisavljevich E, Maxwell AD. For Whom the Bubble Grows: Physical Principles of Bubble Nucleation and Dynamics in Histotripsy Ultrasound Therapy. Ultrasound Med Biol (2019) 45:1056-80. doi: 10.1016/j.ultrasmedbio.2018.10.035

91. Smolock AR, Cristescu MM, Vlaisavljevich E, Gendron-Fitzpatrick A, Green C, Cannata J, et al. Robotically Assisted Sonic Therapy as a Noninvasive Nonthermal Ablation Modality: Proof of Concept in a Porcine Liver Model. Radiology (2018) 287(2):485-93. doi: 10.1148/ radiol.2018171544

92. Vlaisavljevich E, Greve J, Cheng X, Ives K, Shi J, Jin L, et al. Non-Invasive Ultrasound Liver Ablation Using Histotripsy: Chronic Study in an In Vivo Rodent Model. Ultrasound Med Biol (2016) 42(8):1890-902. doi: 10.1016/ j.ultrasmedbio.2016.03.018

93. Vlaisavljevich E, Owens G, Lundt J, Teofilovic D, Ives K, Duryea A, et al. Non-Invasive Liver Ablation Using Histotripsy: Preclinical Safety Study in an In Vivo Porcine Model. Ultrasound Med Biol (2017) 43(6):1237-51. doi: 10.1016/j.ultrasmedbio.2017.01.016

94. Hendricks-Wenger A, Weber P, Simon A, Saunier S, Coutermarsh-Ott S, Grider D, et al. Histotripsy for the Treatment of Cholangiocarcinoma Liver Tumors. In: Vivo Feasibility and Ex Vivo Dosimetry Study. IEEE Transactions on Ultrasonics, Ferroelectrics, and Frequency Control (2021).

95. Lin KW, Kim Y, Maxwell AD, Wang TY, Hall TL, Xu Z, et al. Histotripsy Beyond the Intrinsic Cavitation Threshold Using Very Short Ultrasound Pulses: Microtripsy. IEEE Trans Ultrason Ferroelectr Freq Control (2014) 61 (2):251-65. doi: 10.1109/TUFFC.2014.6722611

96. Vlaisavljevich E, Lin KW, Maxwell A, Warnez M, Mancia L, Singh R, et al. Effects of Ultrasound Frequency and Tissue Stiffness on the Histotripsy Intrinsic Threshold for Cavitation. Ultrasound Med Biol (2015) 41:1651-67. doi: $10.1016 /$ j.ultrasmedbio.2015.01.028

97. Vlaisavljevich E, Kim Y, Allen S, Owens G, Pelletier S, Cain C, et al. ImageGuided non-Invasive Ultrasound Liver Ablation Using Histotripsy: Feasibility Study in an In Vivo Porcine Model. Ultrasound Med Biol (2013) 39(8):1398-409. doi: 10.1016/j.ultrasmedbio.2013.02.005

98. Vlaisavljevich E, Kim Y, Owens G, Roberts W, Cain C, Xu Z. Effects of Tissue Mechanical Properties on Susceptibility to Histotripsy-Induced Tissue Damage. Phys Med Biol (2013) 59(2):253. doi: 10.1088/0031-9155/ $59 / 2 / 253$

99. Vlaisavljevich E, Xu Z, Arvidson A, Jin L, Roberts W, Cain C. Effects of Thermal Preconditioning on Tissue Susceptibility to Histotripsy. Ultrasound Med Biol (2015) 41(11):2938-54. doi: 10.1016/j.ultrasmedbio.2015.07.016

100. Hall TL, Hempel CR, Wojno K, Xu Z, Cain CA, Roberts WW. Histotripsy of the Prostate: Dose Effects in a Chronic Canine Model. Urology (2009) 74 (4):932-7. doi: 10.1016/j.urology.2009.03.049

101. Lake A, Xu Z, Wilkinson J, Cain C, Roberts W. Renal Ablation by Histotripsy -Does it Spare the Collecting System? J Urol (2008) 179(3):1150-4. doi: 10.1016/j.juro.2007.10.033

102. Hempel CR, Hall TL, Cain CA, Fowlkes JB, Xu Z, Roberts WW. Histotripsy Fractionation of Prostate Tissue: Local Effects and Systemic Response in a Canine Model. J Urol (2011) 185(4):1484-9. doi: 10.1016/j.juro.2010.11.044

103. Vlaisavljevich E, Kim Y, Owens G, Roberts W, Cain C, Xu Z. Effects of Tissue Mechanical Properties on Susceptibility to Histotripsy-Induced Tissue Damage. Phys Med Biol (2014) 59(2):253-70. doi: 10.1088/0031-9155/59/ $2 / 253$

104. Vlaisavljevich E, Maxwell A, Warnez M, Johnsen E, Cain C, Xu Z. Histotripsy-Induced Cavitation Cloud Initiation Thresholds in Tissues of 
Different Mechanical Properties. IEEE Trans Ultrasonics Ferroelectrics Frequency Control (2014) 61(2):341-52. doi: 10.1109/TUFFC.2014.6722618

105. Khokhlova VA, Fowlkes JB, Roberts WW, Schade GR, Xu Z, Khokhlova TD, et al. Histotripsy Methods in Mechanical Disintegration of Tissue: Towards Clinical Applications. Int J hyperthermia (2015) 31(2):145-62. doi: 10.3109/ 02656736.2015 .1007538

106. Thanos L, Mylona S, Galani P, Pomoni M, Pomoni A, Koskinas I. Overcoming the Heat-Sink Phenomenon: Successful Radiofrequency Thermal Ablation of Liver Tumors in Contact With Blood Vessels. Diagn Interventional Radiol (2008) 14(1):51.

107. Pillai K, Akhter J, Chua TC, Shehata M, Alzahrani N, Al-Alem I, et al. Heat Sink Effect on Tumor Ablation Characteristics as Observed in Monopolar Radiofrequency, Bipolar Radiofrequency, and Microwave, Using Ex Vivo Calf Liver Model. Medicine (2015) 94(9). doi: 10.1097/MD.00000 00000000580

108. Smolock AR CM, Vlaisavljevich E, Gendron-Fitzpatrick A, Green C, Ziemlewicz TJ and Lee FT. Robotically Assisted Sonic Therapy as a NonInvasive non-Thermal Ablation Modality: Proof of Concept in a Porcine Liver Model. Radiology (2018) 287(2):485-93. doi: 10.1148/radiol.2018171544

109. Coad JE, Kosari K, Humar A, Sielaff TD. Radiofrequency Ablation Causes 'Thermal Fixation'of Hepatocellular Carcinoma: A Post-Liver Transplant Histopathologic Study. Clin Transplant (2003) 17(4):377-84. doi: 10.1034/ j.1399-0012.2003.00062.x

110. Schade GR. Evaluation of the Systemic Response to Boiling Histotripsy Treatment for Renal Carcinoma. (2018). doi: 10.31225/osf.io/qusx8

111. Worlikar T, Mendiratta-Lala M, Greve J, Cho C, Hubbard R, Vlaisavljevich E, et al. Non-Invasive Orthotopic Liver Tumor Ablation Using Histotripsy in an in Vivo Rodent Hepatocellular Carcinoma (Hcc) Model. Soc Interventional Oncology Boston MA USA (2019) 2018:6064-7. doi: 10.1109/EMBC.2018. 8513650

112. Eranki A, Srinivasan P, Ries M, Kim A, Lazarski CA, Rossi CT, et al. HighIntensity Focused Ultrasound (Hifu) Triggers Immune Sensitization of Refractory Murine Neuroblastoma to Checkpoint Inhibitor Therapy. Clin Cancer Res (2020) 26(5):1152-61. doi: 10.1158/1078-0432.CCR-19-1604

113. Hendricks-Wenger A, Sereno J, Gannon J, Zeher A, Brock RM, Betiel-White $\mathrm{N}$, et al. Histotripsy Ablation Alters the Tumor Microenvironment and Promotes Immune System Activation in a Subcutaneous Model of Pancreatic Cancer. IEEE Trans Ultrasonics Ferroelectrics Frequency Control (2021). doi: 10.1109/TUFFC.2021.3078094

114. Worlikar T, Vlaisavljevich E, Gerhardson T, Greve J, Wan S, Kuruvilla S, et al. Histotripsy for Non-Invasive Ablation of Hepatocellular Carcinoma (Hcc) Tumor in a Subcutaneous Xenograft Murine Model. Conf Proc IEEE Eng Med Biol Soc (2018) 2018:6064-7. doi: 10.1109/EMBC.2018.8513650

115. Schade GR, Wang Y-N, D'Andrea S, Hwang JH, Liles WC, Khokhlova TD. Boiling Histotripsy Ablation of Renal Cell Carcinoma in the Eker Rat Promotes a Systemic Inflammatory Response. Ultrasound Med Biol (2019) 45(1):137-47. doi: 10.1016/j.ultrasmedbio.2018.09.006

116. Vlaisavljevich E, Maxwell A, Mancia L, Johnsen E, Cain C, Xu Z. Visualizing the Histotripsy Process: Bubble Cloud-Cancer Cell Interactions in a TissueMimicking Environment. Ultrasound Med Biol (2016) 42:2466-77. doi: 10.1016/j.ultrasmedbio.2016.05.018

117. Zamarron BF, Chen W. Dual Roles of Immune Cells and Their Factors in Cancer Development and Progression. Int J Biol Sci (2011) 7(5):651. doi: 10.7150/ijbs.7.651

118. Fleming V, Hu X, Weber R, Nagibin V, Groth C, Altevogt P, et al. Targeting Myeloid-Derived Suppressor Cells to Bypass Tumor-Induced Immunosuppression. Front Immunol (2018) 9:398. doi: 10.3389/ fimmu.2018.00398

119. Pahk KJ, Shin C-H, Bae IY, Yang Y, Kim S-H, Pahk K, et al. Boiling Histotripsy-induced Partial Mechanical Ablation Modulates Tumour Microenvironment by Promoting Immunogenic Cell Death of Cancers. Sci Rep (2019) 9(1):1-12. doi: 10.1038/s41598-019-45542-z

120. Wang X, Lin Y. Tumor Necrosis Factor and Cancer, Buddies or Foes? 1 Acta Pharmacologica Sin (2008) 29(11):1275-88. doi: 10.1111/j.17457254.2008.00889.x

121. Kratochvill F, Neale G, Haverkamp JM, Van de Velde L-A, Smith AM, Kawauchi D, et al. TNF Counterbalances the Emergence of M2 Tumor Macrophages. Cell Rep (2015) 12(11):1902-14. doi: 10.1016/j.celrep.2015.08.033
122. Dai Z, Wang Z, Lei K, Liao J, Peng Z, Lin M, et al. Irreversible Electroporation Induces CD8+ T Cell Immune Response Against PostAblation Hepatocellular Carcinoma Growth. Cancer Letters (2021) 503:110. doi: 10.1016/j.canlet.2021.01.001

123. Wang D, An G, Xie S, Yao Y, Feng G. The Clinical and Prognostic Significance of CD14+ Hla-DR-/low Myeloid-Derived Suppressor Cells in Hepatocellular Carcinoma Patients Receiving Radiotherapy. Tumor Biol (2016) 37(8):10427-33. doi: 10.1007/s13277-016-4916-2

124. Korbelik M, Banáth J, Saw KM, Zhang W, Čiplys E. Calreticulin as Cancer Treatment Adjuvant: Combination With Photodynamic Therapy and Photodynamic Therapy-Generated Vaccines. Front Oncol (2015) 5:15. doi: 10.3389/fonc. 2015.00015

125. Harris HE, Andersson U. Mini-Review: The Nuclear Protein HMGB1 as a Proinflammatory Mediator. Eur J Immunol (2004) 34(6):1503-12. doi: 10.1002/eji.200424916

126. Pisetsky DS. Cell Death in the Pathogenesis of Immune-Mediated Diseases: The Role of HMGB1 and DAMP-PAMP Complexes. Swiss Med weekly (2011) 141:w13256. doi: 10.4414/smw.2011.13256

127. Chen F, Bao H, Xie H, Tian G, Jiang T. Heat Shock Protein Expression and Autophagy After Incomplete Thermal Ablation and Their Correlation. Int $J$ Hyperthermia (2019) 36(1):95-103. doi: 10.1080/02656736.2018.1536285

128. Fodor P, White B, Khan R. Inflammation-The Role of ATP in Pre-Eclampsia. Microcirculation (2020) 27(1):e12585. doi: 10.1111/micc.12585

129. van Beijnum JR, Buurman WA, Griffioen AW. Convergence and Amplification of Toll-Like Receptor (TLR) and Receptor for Advanced Glycation End Products (RAGE) Signaling Pathways Via High Mobility Group B1 (Hmgb1). Angiogenesis (2008) 11(1):91-9. doi: 10.1007/s10456-008-9093-5

130. Hu Z, Yang XY, Liu Y, Morse MA, Lyerly HK, Clay TM, et al. Release of Endogenous Danger Signals From HIFU-treated Tumor Cells and Their Stimulatory Effects on Apcs. Biochem Biophys Res Commun (2005) 335 (1):124-31. doi: 10.1016/j.bbrc.2005.07.071

131. Gong T, Liu L, Jiang W, Zhou R. DAMP-Sensing Receptors in Sterile Inflammation and Inflammatory Diseases. Nat Rev Immunol (2019) 335:1-18. doi: 10.1038/s41577-019-0215-7

132. Chung AC, Lan HY. Chemokines in Renal Injury. J Am Soc Nephrol (2011) 22(5):802-9. doi: 10.1681/ASN.2010050510

133. Ni L, Lu J. Interferon Gamma in Cancer Immunotherapy. Cancer Med (2018) 7(9):4509-16. doi: 10.1002/cam4.1700

134. Mimura K, Teh JL, Okayama H, Shiraishi K, Kua LF, Koh V, et al. Pd-L1 Expression Is Mainly Regulated by Interferon Gamma Associated With JAKSTAT Pathway in Gastric Cancer. Cancer Sci (2018) 109(1):43-53. doi: $10.1111 /$ cas. 13424

135. Feng L, Qi Q, Wang P, Chen H, Chen Z, Meng Z, et al. Serum Levels of IL-6, Il-8, and IL-10 Are Indicators of Prognosis in Pancreatic Cancer. J Int Med Res (2018) 46(12):5228-36. doi: 10.1177/0300060518800588

136. Shadhu K, Xi C. Inflammation and Pancreatic Cancer: An Updated Review. Saudi J Gastroenterol (2019) 25(1):3-13. doi: 10.4103/sjg.SJG_390_18

137. Lapeyre-Prost A, Terme M, Pernot S, Pointet A-L, Voron T, Tartour E, et al. Immunomodulatory Activity of VEGF in Cancer. Int Rev Cell Mol Biol 330: Elsevier (2017) p:295-342. doi: 10.1016/bs.ircmb.2016.09.007

138. Henry CJ, Ornelles DA, Mitchell LM, Brzoza-Lewis KL, Hiltbold EM. Il-12 Produced by Dendritic Cells Augments CD8+ T Cell Activation Through the Production of the Chemokines CCL1 and CCL17. J Immunol (2008) 181 (12):8576-84. doi: 10.4049/jimmunol.181.12.8576

139. Watford WT, Moriguchi M, Morinobu A, O'Shea JJ. The Biology of IL-12: Coordinating Innate and Adaptive Immune Responses. Cytokine Growth Factor Rev (2003) 14(5):361-8. doi: 10.1016/S1359-6101(03)00043-1

140. Hu Z, Yang XY, Liu Y, Sankin GN, Pua EC, Morse MA, et al. Investigation of HIFU-induced Anti-Tumor Immunity in a Murine Tumor Model. J Trans Med (2007) 5(1):34. doi: 10.1186/1479-5876-5-34

141. Huang X, Yuan F, Liang M, Lo H-W, Shinohara ML, Robertson C, et al. M-HIFU Inhibits Tumor Growth, Suppresses STAT3 Activity and Enhances Tumor Specific Immunity in a Transplant Tumor Model of Prostate Cancer. PloS One (2012) 7(7):e41632. doi: 10.1371/journal.pone.0041632

142. Xing Y, Lu X, Pua EC, Zhong P. The Effect of High Intensity Focused Ultrasound Treatment on Metastases in a Murine Melanoma Model. Biochem Biophys Res Commun (2008) 375(4):645-50. doi: 10.1016/ j.bbrc.2008.08.072 
143. Styn NR, Hall TL, Fowlkes JB, Cain CA, Roberts WW. Histotripsy of Renal Implanted VX-2 Tumor in a Rabbit Model: Investigation of Metastases. Urology (2012) 80(3):724-9. doi: 10.1016/j.urology.2012.06.020

144. Cook JD, Walker CL. The Eker Rat: Establishing a Genetic Paradigm Linking Renal Cell Carcinoma and Uterine Leiomyoma. Curr Mol Med (2004) 4 (8):813-24. doi: 10.2174/1566524043359656

Disclaimer: The content is solely the responsibility of the authors and does not necessarily represent the official views of the NIH or any other funding agency.

Conflict of Interest: EV has financial ties and research collaborations with Histosonics.
The remaining authors declare that the research was conducted in the absence of any commercial or financial relationships that could be construed as a potential conflict of interest.

Copyright $\odot 2021$ Hendricks-Wenger, Hutchison, Vlaisavljevich and Allen. This is an open-access article distributed under the terms of the Creative Commons Attribution License (CC BY). The use, distribution or reproduction in other forums is permitted, provided the original author(s) and the copyright owner(s) are credited and that the original publication in this journal is cited, in accordance with accepted academic practice. No use, distribution or reproduction is permitted which does not comply with these terms. 